\title{
An Improved Shaping Approach to the Preliminary Design of Low-thrust Trajectories
}

\author{
D. M. Novak ${ }^{1}$ and M. Vasile ${ }^{2}$ \\ SpaceART, University of Glasgow, Glasgow G12 8QQ, United Kingdom
}

\begin{abstract}
This paper presents a general framework for the development of shape-based approaches to low-thrust trajectory design. A novel shaping method, based on a three dimensional description of the trajectory in spherical coordinates, is developed within this general framework. Both the exponential sinusoid and the inverse polynomial shaping are demonstrated to be particular two-dimensional cases of the spherical one. The pseudoequinoctial shaping is revisited within the new framework, and the non-osculating nature of the pseudo-equinoctial elements is analyzed. A two step approach is introduced to solve the time of flight constraint, related to the design of low-thrust arcs with boundary constraints for both spherical and pseudo-equinoctial shaping. The solution derived from the shaping approach is improved with a feedback linear-quadratic controller and compared against a direct collocation method based on Finite Elements in Time. The new shaping approach and the combination of shaping and LQ controller are tested on three case studies: a mission to Mars, a mission to asteroid 1989ML, a mission to comet Tempel-1 and a mission to Neptune.
\end{abstract}

\section{Nomenclature}
$\mathbf{A}=$ homogeneous part in the equations of motion
$\mathbf{A}_{\mathrm{g}} \quad=\quad$ submatrix of $\mathbf{A}$
a $=$ acceleration vector of the trajectory parameterized by $t$
$\tilde{\mathbf{a}}=$ acceleration vector of the trajectory parameterized by $s$
$a_{i} \quad=\quad$ coefficients in the shaping function $R$
$\mathbf{B}=$ control matrix coefficient in the equations of motion

\footnotetext{
${ }^{1} \mathrm{PhD}$ candidate, Department of Aerospace Engineering, James Watt South Building, University of Glasgow.

${ }^{2}$ Senior lecturer, Department of Aerospace Engineering, James Watt South Building, University of Glasgow.
} 


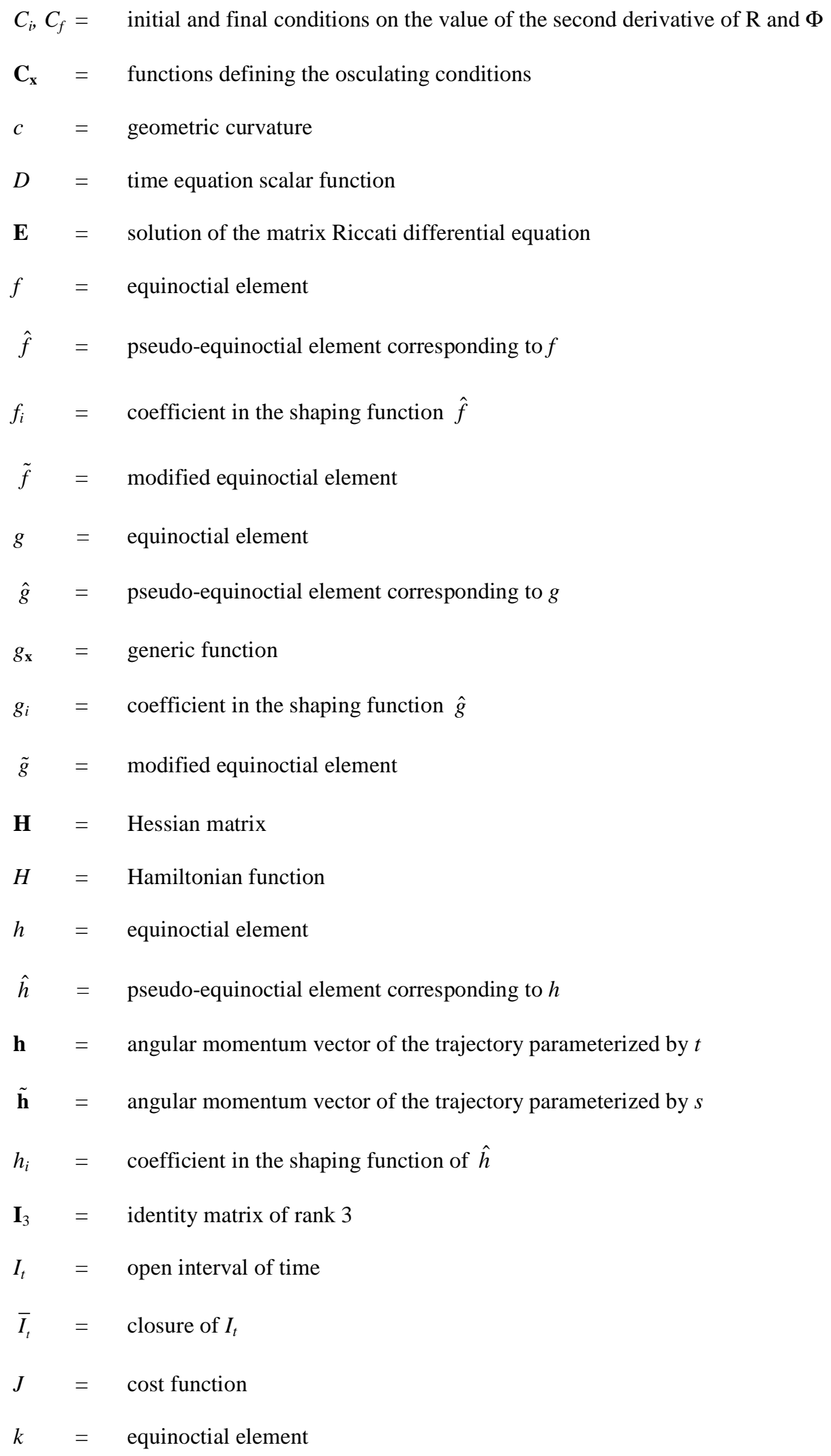




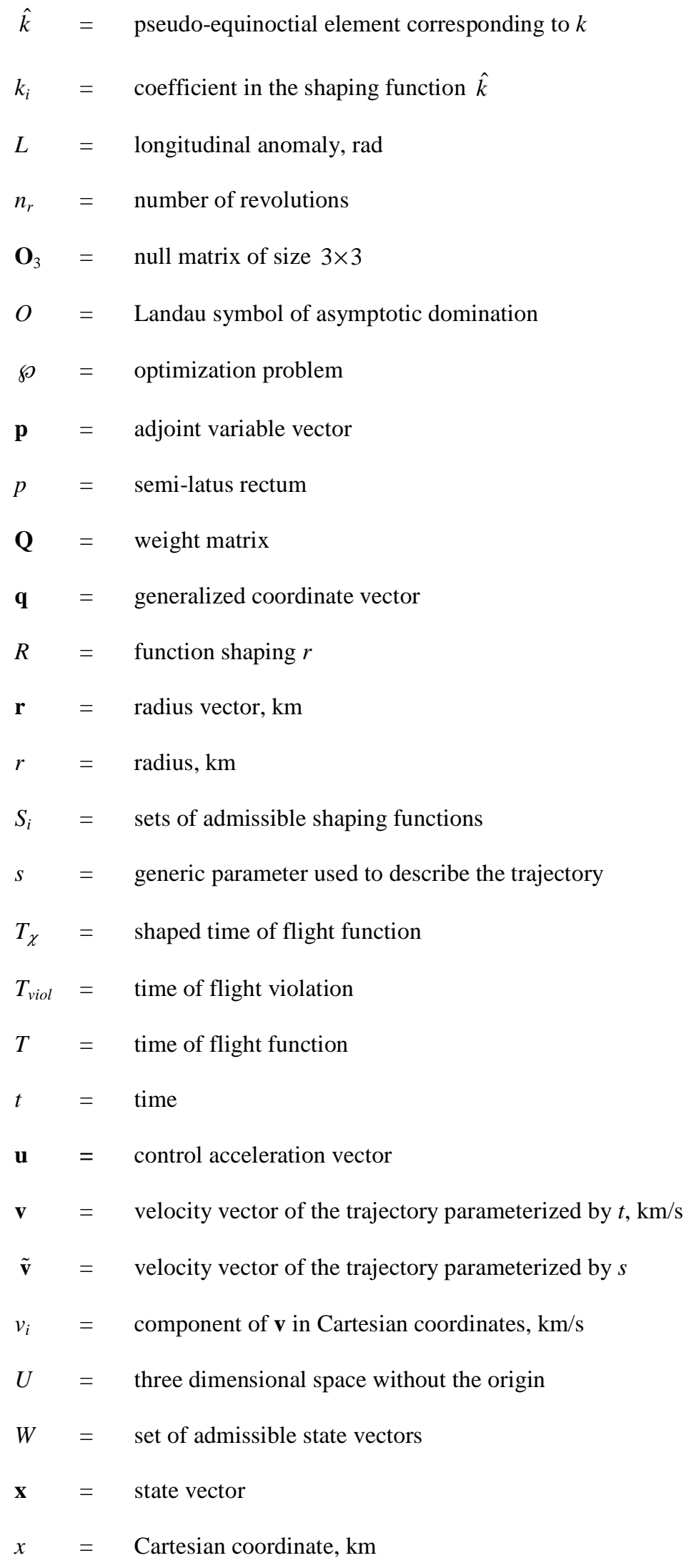


$y=$ Cartesian coordinate, $\mathrm{km}$

$z=$ Cartesian coordinate, $\mathrm{km}$

$\alpha=$ azimuthal angle in the tangential-normal reference frame,rad

$\gamma \quad=\quad$ flight path angle, $\mathrm{rad}$

$\Delta v=$ velocity increment resulting from the control, $\mathrm{km} / \mathrm{s}$

$\delta \quad=\quad$ elevation angle, $\mathrm{rad}$

$\theta=$ azimuthal angle in the spherical reference frame, rad

$\lambda_{i}=$ shaping parameters

$\mu \quad=\quad$ gravitational constant, $\mathrm{km}^{3} / \mathrm{s}^{2}$

$\xi=$ perturbation of the state vector

$v=$ perturbation of the control vector with the linearized equations of motion

$\Phi \quad=\quad$ function shaping $\varphi$

$\varphi=$ elevation angle in the spherical reference frame, rad

$\phi \quad=\quad$ phasing angle, $\mathrm{rad}$

$\chi \quad=\quad$ scalar function shaping $T$

$\Omega_{\mathbf{x}} \quad=\quad$ set on which $\dot{\mathbf{x}}$ is defined

Subscripts

$f \quad=\quad$ final value

$h=$ component along the angular momentum

$i=$ initial value

$l=$ corresponding to the linearized problem

$n=$ component along the normal vector

$\mathbf{r}=$ corresponding to the position components inside the state vector

$r=$ component along the radial vector

real $=$ corresponding to the physical, nonlinearized problem

$t=$ along the velocity vector

$\mathbf{v}=$ corresponding to the velocity components inside the state vector

$\theta=$ component along the transversal vector 
$\varphi=$ component along the base vector corresponding to the elevation

$0=$ corresponding to the reference trajectory

$1=$ corresponding to the augmented linearized system of equations of motion

Superscripts

$T=$ transpose of a matrix

$\square^{\prime} \quad=\quad$ derivative with respect to $s$

$\square^{\prime \prime} \quad=\quad$ double derivative with respect to $s$

$* \quad=$ optimal

\section{Introduction}

$\mathrm{U}_{\mathrm{B}}$ p to now, electric propulsion has been successfully used in a number of missions, both commercial and scientific; remarkable examples are Deep Space 1 [1] and SMART-1 [2]. The savings in propellant mass resulting from the high specific impulse makes this propulsion system attractive for a wide range of high $\Delta \mathrm{v}$ missions. Examples of ongoing or future missions are the NASA Dawn spacecraft, equipped with an ion thruster, that is currently heading towards the asteroid Vesta [3], the Japanese (JAXA) mission Hayabusa to the asteroid Itokawa which carries four electron cyclotron resonance electric engines on board [4] and the European Space Agency (ESA) cornerstone mission BepiColombo that will use solar electric propulsion to propel the spacecraft to Mercury [5]. The potential savings in propellant comes at the price of a more difficult and complex trajectory design. Compared to missions using chemical propulsion, the added difficulty is to optimize continuous thrust profiles instead of optimizing a finite set of impulses. This difference makes analysis of low thrust missions mathematically and computationally more challenging.

During the preliminary assessment of a new mission concept, a large number of scenarios need to be investigated. As a result, the associated search space can be very large. For instance, launch and arrival windows can each span several years. The assessment of different scenarios over a wide range of design parameters requires the efficient generation and evaluation of a large number of feasible trajectories. Finally, common techniques for lowthrust trajectory design [6] require some form of initial guess. However, the generation of suitable initial guess trajectories during the preliminary mission design phase is not trivial. 
In order to make this step more efficient, modeling trajectories analytically has proven to be a viable option. Markopoulos [7] found a class of planar trajectories with a specific expression for the thrust, which he calls Keplerian thrust, with which the trajectory can be expressed analytically while imposing boundary constraints. Markopoulos's results remained however academic and were not applied to the systematic design of transfers. Petropoulos and Longuski [8] proposed to model low-thrust trajectories with exponential sinusoids and obtain the thrust profile from the dynamics, with the aim of designing propellant-optimal low-thrust gravity-assist trajectories. Petropoulos and Longuski's model is planar; the out-of-plane components are only approximated. Moreover the constraints on the total time of flight cannot be satisfied together with the boundary constraints on the velocity vector. However, the exponential sinusoids turned out to provide valuable solutions in the pure point-to-point lowthrust problem. Wall and Conway [9] introduced an inverse polynomial to model the radius of a planar trajectory in polar coordinates, under the assumption of a variable unbounded tangential thrust. The advantage of this approach compared to Petropoulos and Longuski's is the possibility to satisfy all boundary conditions. Later in 2008, Wall extended their approach to cylindrical coordinates [10]. De Pascale and Vasile proposed to shape the variations of the non-singular equinoctial elements due to small perturbations [11]. This shaping approach could model threedimensional trajectories and satisfy boundary, time of flight and thrust constraints. Furthermore, it was demonstrated that the initial guess was good enough to initialize both direct and indirect methods [12].

This paper presents a new approach to quickly generate a large number of suitable initial guess low-thrust transfer trajectories. The method consists of two steps: the fast generation of transfers using a novel shaping method, followed by the improvement of the shaped trajectory by a linear quadratic controller. For the first step, a new shaping approach is proposed that models trajectories in three dimensions using a parameterization in spherical coordinates. The satisfaction of the constraint on the time of flight is treated with a two step approach: a 1dimensional Newton loop over one of the shaping parameters is applied first, if the Newton loop fails, the time evolution is re-shaped such that the constraint is satisfied.

The paper is structured as follows: a general mathematical framework to describe trajectories with shaping methods is presented first; a novel shaping approach is then derived within this framework and tested on a number of cases. The following section presents a linear quadratic (LQ) controller to locally improve the shaped solution, and includes some theoretical results on the optimality of the shaped solution and LQ refinement. The final section demonstrates the effectiveness of the combination of shaping and LQ controller on three test cases: a mission to Mars, a mission to the near Earth asteroid 1989ML, a mission to comet Tempel-1 and a mission to Neptune. 


\section{Low-thrust trajectory shaping}

\section{A. General framework}

The main motivation for the shaping method is to find solutions to a controlled dynamical system, satisfying some conditions on the state vector, by avoiding both the numerical integration of the equations of motion and the solution of an optimal control problem. The underlying idea is to first assign a parameterized shape to the state vector and then compute the control law. This process is normally known as an inverse control problem in aeronautics [13]. In mathematical terms, given the controlled dynamical system:

$$
f:\left\{\begin{array}{l}
W \times \mathbb{R}^{m} \rightarrow \mathbb{R}^{n} \\
(\mathbf{x}, \mathbf{u}) \mapsto \dot{\mathbf{x}}=f(\mathbf{x}, \mathbf{u})
\end{array}\right.
$$

where $m$ is the dimension of $\mathbf{u}, n$ the dimension of $\mathbf{x}$ and $W \subset \mathbb{R}^{n}$, one needs to perform the inverse transformation:

$$
\forall \mathbf{x} \in W, g_{\mathbf{x}}:\left\{\begin{array}{c}
\Omega_{\mathbf{x}} \rightarrow \mathbb{R}^{m} \\
\dot{\mathbf{x}} \mapsto \mathbf{u}=g_{\mathbf{x}}(\dot{\mathbf{x}})
\end{array}\right.
$$

such that the control law $\mathbf{u}$ is obtained as a function of the state vector $\mathbf{x}$ and its derivative $\dot{\mathbf{x}}$. In the following it is assumed that $m<n$ and $f_{\mathbf{x}}$ represents the function $f$ when $\mathbf{x}$ is given. Note that $\mathbf{u} \in \mathbb{R}^{m}$, which means that no constraints on the control are applied at this stage.

One of the main issues when shaping the state vector $\mathbf{x}$ is the definition of the set $\Omega_{\mathbf{x}}$. In fact, a necessary condition to have a physical control vector $\mathbf{u}$ is that the image of $\mathbb{R}^{m}$ under $f_{\mathbf{x}}$ is included in $\Omega_{\mathbf{x}}$, or $\operatorname{Im} f_{\mathbf{x}} \subset \Omega_{\mathbf{x}}$, for all state vectors $\mathbf{x}$, and the corresponding sufficient condition is that the function $g_{\mathbf{x}}$ is defined on $\Omega_{\mathbf{x}}=\operatorname{Im}\left(f_{\mathbf{x}}\right)$. If $g_{\mathbf{x}}$ exists and is defined on $\Omega_{\mathbf{x}}=\operatorname{Im}\left(f_{\mathbf{x}}\right)$ then the composition $g_{\mathbf{x}} \circ f_{\mathbf{x}}$ is bijective (both one-to-one and onto) and therefore $f_{\mathbf{x}}$ is injective (one-to-one) and $g_{\mathbf{x}}$ is surjective (onto). This property means that for the same state vectors, two different control vectors will yield different derivatives of the state vector, which will be easily verified in the scenarios that will be presented later in this paper. In practice, given a coordinate system, a shaping method and a set of dynamic equations, it is required that $f_{\mathbf{x}}$ is surjective, and that $g_{\mathbf{x}}$ is defined on $\operatorname{Im}\left(f_{\mathbf{x}}\right)$. If, furthermore, $g_{\mathbf{x}}$ is bijective, then the control law is unique.

The dynamic equations in this paper are the ones describing the three dimensional motion of a spacecraft subject to the gravitational pull of a central body and to a controllable acceleration. The spacecraft and the central body are assumed to be point masses, with the mass of the spacecraft negligible compared to the one of the planet. The gravity constant of the central mass is denoted by $\mu$. No assumption is made on the propulsion system: the thrust 
magnitude and the propellant consumption are obtained from the control law once an initial spacecraft mass and a value for the specific impulse are provided.

Independent of whether Cartesian, spherical coordinates, Keplerian or Equinoctial elements are used to parameterize the motion of the spacecraft, the equations of motion around the central body can be expressed as:

$$
\dot{\mathbf{x}}=\mathbf{A}(\mathbf{x})+\mathbf{B}(\mathbf{x}) \mathbf{u}
$$

With the number of states $n=6$ and the number of controls $m=3$. The space in which the state vectors $\mathbf{x}$ are defined, $W$, is an open subset of $\mathbb{R}^{6}$. Note that $f_{\mathbf{x}}$ in this case is affine. The physics of the problem are such that each of the three components of the control vector has an effect on the state vector, thus $f_{x}$ is injective. $\operatorname{Im}\left(f_{\mathbf{x}}\right)$ is a three dimensional manifold of $\mathbb{R}^{6}$, which can be defined by three equations of the type $\mathbf{C}_{\mathbf{x}}(\dot{\mathbf{x}})=0$. A general expression for $g_{\mathbf{x}}$ can be given by:

$$
\mathbf{u}=\left[\mathbf{B}(\mathbf{x})^{T} \mathbf{B}(\mathbf{x})\right]^{-1} \mathbf{B}(\mathbf{x})^{T}[\dot{\mathbf{x}}-\mathbf{A}(\mathbf{x})]
$$

Note that $\left[\mathbf{B}(\mathbf{x})^{T} \mathbf{B}(\mathbf{x})\right]^{-1} \mathbf{B}(\mathbf{x})^{T}$ is the Moore-Penrose pseudo-inverse of $\mathbf{B}(\mathbf{x})$. It will be shown that for all $\mathbf{x} \in W, \mathbf{B}(\mathbf{x})^{T} \mathbf{B}(\mathbf{x})$ is invertible and $g_{\mathbf{x}}$ is surjective since $g_{\mathbf{x}}$ is affine and each component of the control vector will be influenced by at least one component of $\dot{\mathbf{x}}$.

Finally, the particular type of dynamical system that will be considered in this paper is described by a set of three second-order differential equations. In that case, three independent coordinates $\mathbf{q}$ and their respective derivatives $\dot{\mathbf{q}}$ define the state vector $\mathbf{x}=[\mathbf{q}, \dot{\mathbf{q}}]^{T}$. The vector $\mathbf{q}$ represents the state of the spacecraft in the configuration space expressed either in Cartesian or spherical coordinates.

Let us assume that the trajectory is parameterized by a variable $s$, defined on a closed interval $\left[s_{i} s_{f}\right]$ and that there is a smooth mapping between the time $t$ and $s$. Denoting by a prime the derivatives with respect to $s$, one would have the relationship $\dot{s}(s)=1 / t^{\prime}(s)$ and $\ddot{s}(s)=-t^{\prime \prime}(s) /\left(t^{\prime}(s)\right)^{3}$. Hence, the state vector becomes $\mathbf{x}=\left[\mathbf{q}, \dot{s} \mathbf{q}^{\prime}\right]^{T}$ and the dynamical equations can be written as:

$$
\dot{s}^{2} \mathbf{q}^{\prime \prime}+\ddot{s} \mathbf{q}^{\prime}=\mathbf{A}\left(\mathbf{q}, \dot{s} \mathbf{q}^{\prime}\right)+\mathbf{B}\left(\mathbf{q}, \dot{s} \mathbf{q}^{\prime}\right) \mathbf{u}
$$

This kind of reparameterization was first used by Baumgarte in 1972 [14] for the purpose of stabilizing the numerical integration of equations of motion. The general formulation in Eqs. (4) and (5) will be translated into two specific shaping approaches in spherical coordinates and Cartesian coordinates.

\section{B. Spherical Shaping}


The trajectory of the spacecraft in the three dimensional space is defined by the spherical coordinates,

$$
(r, \theta, \varphi) \in \mathbb{R}^{+} \times \frac{\mathbb{R}}{2 \pi \mathbb{Z}} \times\left(\frac{-\pi}{2}+\frac{\mathbb{R}}{\pi \mathbb{Z}}\right)
$$

where $r$ is the distance from the central body, $\theta$ is the azimuthal angle and $\varphi$ is the elevation angle (see Fig. 1). If the variation of the position is taken with respect to time, the state vector is $[r, \theta, \varphi, \dot{r}, \dot{\theta}, \dot{\varphi}]^{T}$.

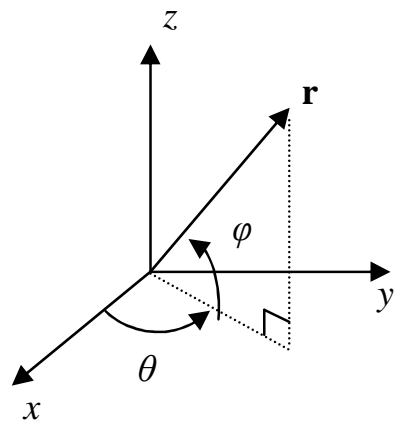

Fig. 1 Illustration of the spherical coordinate system

If, instead, the angle $\theta$ is taken as the parameter $s$ to parameterize the trajectory, then $r=R(\theta), \varphi=\Phi(\theta)$ and $t=T(\theta)$. The transformation between $t$ and $\theta$ holds if there is a smooth one-to-one mapping between $t$ and $\theta$, which implies that $\theta$ is strictly monotonous with respect to time. The state vector becomes $\mathbf{x}=\left[r, t, \varphi, r^{\prime}, t^{\prime}, \varphi^{\prime}\right]^{T}$ where the prime represents the derivative with respect to $\theta$. This parameterization is non-singular if the poles and the origin are excluded from the set of admissible positions. Moreover, the angle $\theta$ will account for the $n_{r}$ revolutions of the trajectory. Hence, the space $W$ is defined as $W=\mathbb{R}_{+}^{*} \times\left[\begin{array}{lll}\theta_{i} & \theta_{f}+2 n_{r} \pi\end{array}\right] \times\left(\begin{array}{ll}-\pi / 2 & \pi / 2\end{array}\right) \times \mathbb{R}^{3}$. The equations of motion in an inertial reference frame are:

$$
\frac{d^{2} \mathbf{r}}{d t^{2}}=-\mu \frac{\mathbf{r}}{r^{3}}+\mathbf{u}
$$

where the position vector is $\mathbf{r}=[r \cos \theta \cos \varphi, r \sin \theta \cos \varphi, r \sin \varphi]^{T}$. Since the position vector is parametrized with $\theta$, then:

$$
\dot{\theta}^{2} \frac{d^{2} \mathbf{r}}{d \theta^{2}}+\ddot{\theta} \frac{d \mathbf{r}}{d \theta}=-\mu \frac{\mathbf{r}}{r^{3}}+\mathbf{u}
$$

with $\dot{\theta}=1 / t^{\prime}$ and $\ddot{\theta}=-t^{\prime \prime} / t^{\prime 3}$. Expanding Eq. (7), one can obtain the dynamical system for $\mathbf{x}$ as in Eq. (5). Finally, the control vector is obtained straight from Eq. (7), after having inserted the expression of $\mathbf{r}$ as a function of the spherical coordinates. 
If the geometrical trajectory is given, then one still needs to set the evolution of the spacecraft along that trajectory in order to define completely the physical transfer. This is done by providing $\dot{\theta}$ as a function of $\theta$. Then one can have $\ddot{\theta}$, and through (7), the control profile $\mathbf{u}$ is extracted. Therefore, if the shape of a trajectory is fixed, then only one degree of freedom remains to fix the transfer. However, it is not straightforward to set the time evolution a priori, such that the final control profile is systematically close to optimal.

A simple relationship is established here between $\dot{\theta}$ and the normal component of $\mathbf{u}$, in the tangential-normalout-of-plane frame. This relationship turns out to be useful to set a 'shape' for the time evolution based on reasonable considerations.

The velocity vector $\mathbf{v}$ is expressed as:

$$
\mathbf{v}=\frac{d \mathbf{r}}{d t}=\dot{\theta} \frac{d \mathbf{r}}{d \theta}
$$

and the acceleration vector $\mathbf{a}$ as:

$$
\mathbf{a}=\frac{d \mathbf{v}}{d t}=\ddot{\theta} \frac{d \mathbf{r}}{d \theta}+\dot{\theta}^{2} \frac{d^{2} \mathbf{r}}{d \theta^{2}}
$$

In the following, the vectors $\frac{d \mathbf{r}}{d \boldsymbol{\theta}}$ and $\frac{d^{2} \mathbf{r}}{d \boldsymbol{\theta}^{2}}$ will be called $\tilde{\mathbf{v}}$ and $\tilde{\mathbf{a}}$ respectively. They are entirely described by the geometry of the trajectory and therefore by $r, \varphi$ and their first and second derivatives. The vector $\tilde{\mathbf{h}}=\mathbf{r} \wedge \tilde{\mathbf{v}}$ is also introduced, its magnitude is noted $\tilde{h}$.

The unit vectors $\left(\mathbf{e}_{t}, \mathbf{e}_{n}, \mathbf{e}_{h}\right)$ defining the tangential-normal-out-of-plane reference frame are introduced and the equations of motion are projected onto it:

$$
\mathbf{u}=\left|\begin{array}{l|l}
u_{t} \\
u_{n} \\
u_{h}
\end{array}\right| \begin{gathered}
\frac{\mu}{r^{2}} \mathbf{e}_{r} \cdot \mathbf{e}_{t}+\ddot{\theta} \tilde{\mathbf{v}} \cdot \mathbf{e}_{t}+\dot{\theta}^{2} \tilde{\mathbf{a}} \cdot \mathbf{e}_{t} \\
\frac{\mu}{r^{2}} \mathbf{e}_{r} \cdot \mathbf{e}_{n}+\dot{\theta}^{2} \tilde{\mathbf{a}} \cdot \mathbf{e}_{n} \\
\dot{\theta}^{2} \tilde{\mathbf{a}} \cdot \mathbf{e}_{h}
\end{gathered}
$$

The second and the third component of $\mathbf{u}$ in (10) are of interest because they only involve $\dot{\theta}^{2}$ while $\ddot{\theta}$ is absent. The projection on the out-of-plane component does not provide much information. However the normal component of $\mathbf{u}$ can be rewritten as: 


$$
u_{n}=\frac{\mu}{r^{2}} \mathbf{e}_{r} \cdot \mathbf{e}_{n}+\dot{\theta}^{2} \frac{\tilde{\mathbf{a}} \cdot(\tilde{\mathbf{h}} \wedge \tilde{\mathbf{v}})}{\tilde{h} \tilde{v}}
$$

Since $\mathbf{e}_{r} \cdot \mathbf{e}_{n}=\cos \gamma$ and $\tilde{h}=r \tilde{v} \cos \gamma$, one obtains:

$$
D \dot{\theta}^{2}=\frac{\mu}{r^{2}}+\frac{u_{n}}{\cos \gamma}
$$

where:

$$
D=\frac{r}{\tilde{h}^{2}} \tilde{\mathbf{a}} \cdot(\tilde{\mathbf{h}} \wedge \tilde{\mathbf{v}})
$$

This expression depends uniquely on $R, R^{\prime}, R^{\prime},, \Phi, \Phi^{\prime}$ and $\Phi^{\prime}$, so on the pure geometrical shape of the trajectory. Finally, if one uses the physical velocity $\mathbf{v}$, acceleration $\mathbf{a}$ and angular momentum $\mathbf{h}$, then:

$$
\begin{aligned}
\mathbf{a} \cdot(\mathbf{h} \wedge \mathbf{v}) & =\left(\ddot{\theta} \tilde{\mathbf{v}}+\dot{\theta}^{2} \tilde{\mathbf{a}}\right) \cdot(\dot{\theta} \tilde{\mathbf{h}} \wedge \dot{\theta} \tilde{\mathbf{v}}) \\
& =\dot{\theta}^{4} \tilde{\mathbf{a}} \cdot(\tilde{\mathbf{h}} \wedge \tilde{\mathbf{v}})
\end{aligned}
$$

and $D$ can then be rewritten as:

$$
D=\frac{r}{\dot{\theta}^{2} h^{2}} \mathbf{a} \cdot(\mathbf{h} \wedge \mathbf{v})
$$

The quantity $D$ has the sign of $\mathbf{a} \cdot(\mathbf{h} \wedge \mathbf{v})$, which is the same as the sign of $\tilde{\mathbf{a}} \cdot(\tilde{\mathbf{h}} \wedge \tilde{\mathbf{v}})$, thus $D$ is positive when the trajectory is curved towards the central body.

The scalar $D$ is independent of the reference frame, and can be expressed using the components of the position, velocity and acceleration in any one reference frame. Using the radial-orthoradial-out-of-plane coordinate system $\left(\mathbf{e}_{r}, \mathbf{e}_{o}, \mathbf{e}_{h}\right)$, one has $\tilde{v}_{h}=0$, such that $D=\frac{\tilde{v}_{r} \tilde{a}_{o}-\tilde{v}_{o} \tilde{a}_{r}}{\tilde{v}_{o}}$.

It is shown in Appendix A that $\tilde{\mathbf{v}}$ and $\tilde{\mathbf{a}}$ are written in $\left(\mathbf{e}_{r}, \mathbf{e}_{o}, \mathbf{e}_{h}\right)$ as

$$
\tilde{\mathbf{v}}=\mid \begin{array}{l|c}
\tilde{v}_{r} & r^{\prime} \\
\tilde{v}_{o} & r \sqrt{\varphi^{\prime 2}+\cos ^{2} \varphi} \\
\tilde{v}_{h} & 0
\end{array}
$$




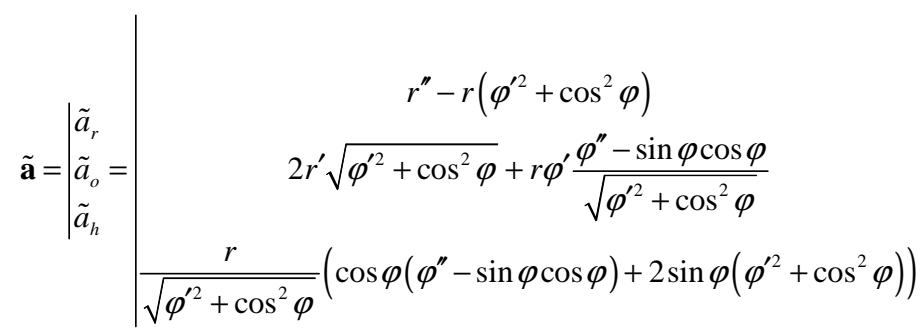

The expression for $D$ becomes then:

$$
D=-r^{\prime \prime}+2 \frac{r^{\prime 2}}{r}+r^{\prime} \varphi^{\prime} \frac{\varphi^{\prime \prime}-\sin \varphi \cos \varphi}{\varphi^{\prime 2}+\cos ^{2} \varphi}+r\left(\varphi^{\prime 2}+\cos ^{2} \varphi\right)
$$

The functions $R, \Phi$ and $T$ are the respective functions that shape $r, \varphi$ and $t$; the corresponding control profile can be obtained, along with the $\Delta v$ and propellant consumption, given the spacecraft initial mass and specific impulse. Functions $R$ and $\Phi$ model the pure geometry of the trajectory, while the function $T$ shapes the time evolution along the trajectory. It is assumed that the shaping functions $R, \Phi$ and $T$ belong to sets of admissible functions $S_{R}, S_{\Phi}$ and $S_{T}$ that are twice continuously differentiable. Now, if the function $T$, defined through its derivative:

$$
T^{\prime}=\sqrt{\frac{D R^{2}}{\mu}}
$$

is used to shape $t$, then by plugging it into (12), one finds that the control vector corresponding to the geometrical trajectory defined by $R$ and $\Phi$ will have no component normal to the tangential plane of the trajectory (i.e. $u_{n}=0$ ). Note that shaping of the derivative $T^{\prime}$ instead of $T$ is not an issue because the origin of time can be set as an additive constant to $T$. Eq. (19) requires the condition $D>0$ in order to have a real time of flight. Geometrically speaking, the latter condition means that the plane defined by $\mathbf{v}$ and $\mathbf{h}$ (or in other words by the admissible control vectors) divides the space in two, and the trajectory must be curved towards the half-space containing the center of gravity. In fact, if the acceleration vector pointed towards the opposite half-space, a control component outside of the separating plane would be required to balance the gravitational pull of the central body, therefore $u_{n} \neq 0$ (see Fig. 2). 


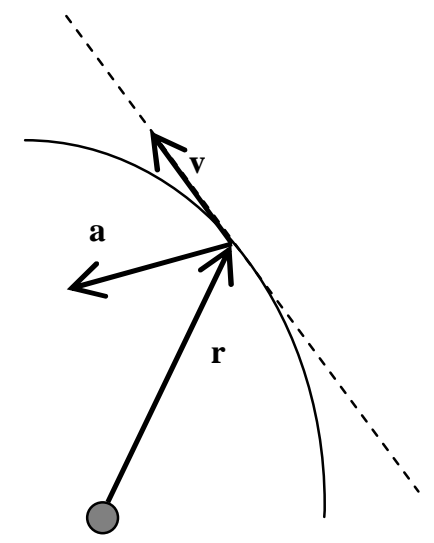

Fig. 2 Illustration in $2 D$ of the condition $D>0$. The trajectory is the arc, the velocity vector is aligned with the tangent (dashed line).

In the particular case of a two dimensional trajectory, one obtains:

$$
D=\frac{c R^{2}}{\cos ^{3} \gamma}
$$

where $c$ is the algebraic curvature of the trajectory, thus $D>0$ if the curvature $c$ is positive. The time of flight and $\Delta v$ corresponding to the shaped trajectory are obtained by integrating respectively $T^{\prime}$ and $|\mathbf{u}| T^{\prime}$ over the interval $\left[\begin{array}{ll}\theta_{i} & \theta_{f}+2 n_{r} \pi\end{array}\right]$.

It should be noted that by shaping the time evolution $T$ with the expression in Eq. (19), $R$ and $\Phi$ define completely $T^{\prime}$ and the time of flight $T\left(\theta_{f}\right)-T\left(\theta_{i}\right)$. This method can be problematic when a constraint on the time of flight exists. However it is generally difficult to find a priori a shaping function $T$ in such a way to obtain a control that is near optimal, i.e., interesting in practice. Indeed, if $T$ was a completely arbitrary function, then the forces required to keep the spacecraft on the path prescribed by $R$ and $\Phi$ can have the same or a higher order of magnitude than the one of the gravitational pull, in which case the transfer cannot be qualified as low-thrust anymore. Using an expression for $T^{\prime}$ as in (19) will result in reasonable thrust profiles, especially when an efficient change in the semi-major axis is sought for.

$R$ and $\Phi$ can be in any function space such that $R>0$ and $-\pi / 2<\Phi<\pi / 2$. It is judicious, however, to choose expressions for which the boundary constraints on the position and velocity can be computed analytically. Having the flexibility to impose boundary constraints for initial guesses both on position and velocity helps to improve the convergence of optimizers and more importantly it helps to generate feasible solutions that provide a good estimation of the required thrust level, time of flight and propellant consumption. The boundary conditions are: 


$$
\begin{cases}R\left(\theta_{i}\right)=R_{i} & R\left(\theta_{f}+2 \mathrm{n}_{r} \pi\right)=R_{f} \\ \Phi\left(\theta_{i}\right)=\Phi_{i} & \Phi\left(\theta_{f}+2 \mathrm{n}_{r} \pi\right)=\Phi_{f} \\ T^{\prime}\left(\theta_{i}\right)=\frac{R_{i} \cos \Phi_{i}}{v_{\theta i}} & T^{\prime}\left(\theta_{f}+2 \mathrm{n}_{r} \pi\right)=\frac{R_{f} \cos \Phi_{f}}{v_{\theta f}} \\ R^{\prime}\left(\theta_{i}\right)=v_{r i} & R^{\prime}\left(\theta_{f}+2 \mathrm{n}_{r} \pi\right)=v_{r f} \\ \Phi^{\prime}\left(\theta_{i}\right)=\frac{v_{\varphi i}}{R_{i}} & \Phi^{\prime}\left(\theta_{f}+2 \mathrm{n}_{r} \pi\right)=\frac{v_{\varphi f}}{R_{f}}\end{cases}
$$

The boundary conditions on $T^{\prime}$ are expressed as the boundary conditions on $R^{\prime \prime}$ and $\Phi^{\prime \prime}$ :

$$
\left\{\begin{array}{c}
R^{\prime \prime}\left(\theta_{i}\right)+\alpha_{i} \Phi^{\prime \prime}\left(\theta_{i}\right)=C_{i} \\
R^{\prime \prime}\left(\theta_{f}\right)+\alpha_{f} \Phi^{\prime \prime}\left(\theta_{f}\right)=C_{f}
\end{array}\right.
$$

Where

$$
\begin{aligned}
\alpha_{i / f} & =-\frac{R^{\prime} \Phi^{\prime}\left(\theta_{i / f}\right)}{\Phi^{\prime 2}\left(\theta_{i / f}\right)+\cos ^{2} \Phi\left(\theta_{i / f}\right)} \\
& =-\frac{v_{r i f f} v_{\varphi i f f}}{R_{i / f}\left(v_{\varphi i / f}^{2} / R_{i / f}^{2}+\cos ^{2} \Phi_{i / f}\right)}
\end{aligned}
$$

and

$$
\begin{aligned}
C_{i / f} & =-\frac{\mu T^{\prime 2}\left(\theta_{i / f}\right)}{R_{i / f}^{2}}+2 \frac{R_{i / f}^{\prime 2}}{R_{i / f}}+R_{i / f}\left(\Phi^{\prime 2}\left(\theta_{i / f}\right)+\cos ^{2} \Phi\left(\theta_{i / f}\right)\right)-R_{i / f}^{\prime} \Phi^{\prime}\left(\theta_{i / f}\right) \frac{\sin \Phi\left(\theta_{i / f}\right) \cos \Phi\left(\theta_{i / f}\right)}{\Phi^{\prime 2}\left(\theta_{i / f}\right)+\cos ^{2} \Phi\left(\theta_{i / f}\right)} \\
& =-\frac{\mu \cos ^{2} \Phi_{i / f}}{v_{i i / f}^{2}}+2 \frac{v_{r i f f}^{2}}{R_{i / f}}+R_{i / f}\left(\frac{v_{\varphi i l f}^{2}}{R_{i / f}^{2}}+\cos ^{2} \Phi_{i / f}\right)-\frac{v_{r i / f} v_{\varphi i / f}}{R_{i / f}} \frac{\sin \Phi_{i / f} \cos \Phi_{i / f}}{\frac{v_{\varphi i / f}^{2}}{R_{i / f}^{2}}+\cos ^{2} \Phi_{i / f}}
\end{aligned}
$$

There are therefore ten boundary conditions: four on the radius, four on the elevation angle and two combined between the two. Thus, the functions $R$ and $\Phi$ must have at least 10 free parameters to satisfy the boundary conditions. If there are exactly 10 parameters, the time of flight is uniquely defined.

A relatively wide set of shaping functions for $R$ and $\Phi$ can be used such that the boundary conditions can be satisfied analytically. The boundary conditions expressed in (21) and (22) suggest that functions of the form $R(\theta)=\sum_{k=1}^{n} a_{k} R_{k}(\theta)$ and $\Phi(\theta)=\sum_{k=1}^{m} b_{k} \Phi_{k}(\theta)$ are interesting because their derivatives remain linear combinations of the unknown coefficients and therefore the boundary conditions can be solved by inverting a 10 by 10 matrix. Note that one needs to have $n \geq 4, m \geq 4$ and $n+m=10$. 
However there is a wider set of functions that allow satisfying constraints in such a way. If one considers those functions that can be written as $R(\theta)=R_{0}\left(\sum_{k=1}^{n} a_{k} R_{k}(\theta)\right)$ and $\Phi(\theta)=\Phi_{0}\left(\sum_{i=1}^{m} b_{k} \Phi_{k}(\theta)\right)$ where $R_{0}$ and $\Phi_{0}$ are functions that can be analytically inversed, then the boundary conditions on $R$ and $\Phi$ need to be rewritten as $\sum_{k=1}^{n} a_{k} R_{k}\left(\theta_{i / f}\right)=R_{0}^{-1}\left(R_{i / f}\right)$ and $\sum_{i=1}^{m} b_{k} \Phi_{k}\left(\theta_{i / f}\right)=\Phi_{0}^{-1}\left(\Phi_{i / f}\right)$, those on $R^{\prime}$ and $\Phi^{\prime}$ become:

$$
\begin{aligned}
& \sum_{k=1}^{n} a_{k} R_{k}^{\prime}\left(\theta_{i / f}\right)=\frac{v_{r i f f}}{R_{0}^{\prime}\left(\sum_{k=1}^{n} a_{k} R_{k}\left(\theta_{i / f}\right)\right)}=\frac{v_{r i / f}}{R_{0}^{\prime}\left(R_{0}^{-1}\left(R_{i / f}\right)\right)} \\
& \sum_{i=1}^{m} b_{k} \Phi_{k}^{\prime}\left(\theta_{i / f}\right)=\frac{v_{\varphi i / f} / R_{i / f}}{\Phi_{0}^{\prime}\left(\sum_{i=1}^{m} b_{k} \Phi_{k}\left(\theta_{i / f}\right)\right)}=\frac{v_{\varphi i / f} / R_{i / f}}{\Phi_{0}^{\prime}\left(\Phi_{0}^{-1}\left(\Phi_{i / f}\right)\right)}
\end{aligned}
$$

Finally the boundary conditions on $T^{\prime}$ in (22), where the second derivatives of $R$ and $\Phi$ are present, become

$$
\begin{aligned}
& \sum_{k=1}^{n} a_{k} R_{k}^{\prime \prime}\left(\theta_{i / f}\right) \cdot R_{0}^{\prime}\left(\sum_{k=1}^{n} a_{k} R_{k}\left(\theta_{i / f}\right)\right)+\left(\sum_{k=1}^{n} a_{k} R_{k}^{\prime}\left(\theta_{i / f}\right)\right)^{2} \cdot R_{0}^{\prime \prime}\left(\sum_{k=1}^{n} a_{k} R_{k}\left(\theta_{i / f}\right)\right) \\
& +\alpha_{i / f}\left(\sum_{i=1}^{m} b_{k} \Phi_{k}^{\prime \prime}\left(\theta_{i / f}\right) \cdot \Phi_{0}^{\prime}\left(\sum_{i=1}^{m} b_{k} \Phi_{k}\left(\theta_{i / f}\right)\right)+\left(\sum_{i=1}^{m} b_{k} \Phi_{k}^{\prime}\left(\theta_{i / f}\right)\right)^{2} \cdot \Phi_{0}^{\prime \prime}\left(\sum_{i=1}^{m} b_{k} \Phi_{k}\left(\theta_{i / f}\right)\right)\right)=C_{i / f}
\end{aligned}
$$

And after rearranging, the following linear equations in $\left(a_{k}\right)$ and $\left(b_{k}\right)$ are obtained:

$$
\begin{aligned}
& \sum_{k=1}^{n} a_{k} R_{k}{ }^{\prime \prime}\left(\theta_{i / f}\right) \cdot R_{0}{ }^{\prime}\left(R_{0}^{-1}\left(R_{i / f}\right)\right)+\alpha_{i / f} \sum_{i=1}^{m} b_{k} \Phi_{k}{ }^{\prime \prime}\left(\theta_{i / f}\right) \cdot \Phi_{0}{ }^{\prime}\left(\Phi_{0}^{-1}\left(\Phi_{i / f}\right)\right)= \\
& =C_{i / f}-\frac{v_{r i / f}^{2}}{R_{0}^{\prime}\left(R_{0}^{-1}\left(R_{i / f}\right)\right)^{2}} \cdot R_{0}{ }^{\prime \prime}\left(R_{0}^{-1}\left(R_{i / f}\right)\right)-\alpha_{i / f} \frac{v_{\varphi i / f}^{2} / R_{i / f}^{2}}{\Phi_{0}{ }^{\prime}\left(\Phi_{0}^{-1}\left(\Phi_{i / f}\right)\right)^{2}} \cdot \Phi_{0}{ }^{\prime \prime}\left(\Phi_{0}^{-1}\left(\Phi_{i / f}\right)\right)
\end{aligned}
$$

Therefore one has a relatively wide array of possibilities for the shaping functions and boundary conditions on position and velocity can be satisfied by inverting a 10 by 10 matrix.

In the test cases in this paper, $S_{R}$ is the set of functions expressed in a form that is reminiscent of the expression of the radius in Keplerian elements, and $S_{\Phi}$ is such that $\Phi$ oscillates:

$$
\left\{\begin{array}{l}
R=\frac{1}{a_{0}+a_{1} \theta+a_{2} \theta^{2}+\left(a_{3}+a_{4} \theta\right) \cos \theta+\left(a_{5}+a_{6} \theta\right) \sin \theta} \\
\Phi=\left(b_{0}+b_{1} \theta\right) \cos \theta+\left(b_{2}+b_{3} \theta\right) \sin \theta
\end{array}\right.
$$

The motivation for this choice is that the minimum-thrust arc is the Keplerian arc. No singularity was encountered for $R$ in the test cases of this paper, the value for the radius remained strictly positive. In the same way, $\Phi$ always remained within the interval $(-\pi / 2 ; \pi / 2)$. Note that, in Eq. (28), the total number of free parameters is 11 
and not 10. The extra parameter can be used as an additional degree of freedom to modify the shape of the trajectory. It can, for instance, be used to satisfy a constraint on the time of flight, as described in Section II.D.

Finally, it is shown that the shaping approaches of Petropoulos and Longuski, and Wall and Conway are special cases of the general three dimensional spherical shaping. In 2000 Petropoulos and Longuski [17] proposed the use of a two dimensional shape, expressed in polar coordinates, for designing low-thrust trajectories with gravity-assists. The radius takes the following form:

$$
R(\theta)=k_{0} \exp \left[k_{1} \sin \left(k_{2} \theta+\phi\right)\right]
$$

A tangential thrust is assumed along the trajectory and, according to the theory developed for the three dimensional spherical model, tangential thrust is possible only if the quantity $D$ defined in Eq. (15) is strictly positive. In two dimensions, Eq. (18) translates into the expression $D=-R^{\prime \prime}+R+2 R^{\prime 2} / R=\left[R^{-1}+\left(R^{-1}\right)^{\prime \prime}\right] /\left(R^{-1}\right)^{2}$. One easily finds that tangential thrust is possible if and only if $\left|k_{1}\right| k_{2}^{2}<1$, as derived by Petropoulos and Longuski.

Petropoulos and Longuski found solutions to the two-point boundary value problem by tuning the value of $k_{2}$ such that the spiral intersects the target orbit at the right time, without necessarily matching the velocities at the boundaries [18]. Izzo [19] studied the Lambert problem for the exponential sinusoids and found that it could be solved for certain ranges of time of flight that depend on the initial flight path angles. Therefore, this method cannot satisfy all possible boundary conditions on position and velocity without an additional impulsive $\Delta v$.

Wall and Conway [9] devised a shape-based method similar to that of Petropoulos and Longuski, with the difference that they used the inverse of a $6^{\text {th }}$ degree polynomial to model the radius instead of an exponential sinusoid. Their expression of $R$ contains more free parameters such that the boundary conditions on both position and velocity can be accommodated. An additional parameter in $R$ is used to satisfy the time of flight constraints. The expression used for $R$ suffers from two drawbacks though. The first is that it does not cover the unperturbed Keplerian motion. The second is that the $6^{\text {th }}$ degree polynomial in the expression of $R$ can have at most 5 extrema. Therefore, if one models a transfer between two elliptical orbits using 3 revolutions or more, the inverse polynomials would not be able to model oscillations of the radii between successive pericenters and apocenters.

\section{Shaping the pseudo-equinoctial elements}

In 2006, De Pascale and Vasile proposed a different shaping approach based on the variation of the orbital elements [11]. Their shaping approach makes use of a set of pseudo-equinoctial elements to shape the Cartesian coordinates. Here, the pseudo-equinoctial shaping is revisited in the general framework laid out in Section II.A. The 
equations of motion used to calculate the control vector are the same as Eqs. (7). The expression of the equinoctial elements with respect to the Keplerian elements $\left(\begin{array}{llllll}a & e & i & \Omega & \omega & v\end{array}\right)$ is reminded here:

$$
\begin{aligned}
p & =a\left(1-e^{2}\right) \\
f & =e \cos \omega \\
g & =e \sin \omega \\
h & =\tan \frac{i}{2} \cos \Omega \\
k & =\tan \frac{i}{2} \sin \Omega \\
L & =\Omega+\omega+v
\end{aligned}
$$

The longitudinal anomaly $L$ is used as parameter $s$ instead of the azimuthal angle $\theta$. The state vector is defined as $\mathbf{x}=[p, f, g, h, k, t]^{T}$ and one can obtain the Cartesian position vector from the transformation [11]:

$$
\mathbf{r}(\mathbf{x}, L)=\mid \begin{gathered}
\frac{p\left[\left(1+h^{2}-k^{2}\right) \cos L+2 h k \sin L\right]}{(1+f \cos L+g \sin L)\left(1+h^{2}+k^{2}\right)} \\
\frac{p\left[\left(1-h^{2}+k^{2}\right) \sin L+2 h k \cos L\right]}{(1+f \cos L+g \sin L)\left(1+h^{2}+k^{2}\right)} \\
\frac{2 p(h \sin L-k \cos L)}{(1+f \cos L+g \sin L)\left(1+h^{2}+k^{2}\right)}
\end{gathered}
$$

A trajectory can be described as a continuous succession of points, parameterized by $L$ here, where each point is on an instantaneous ellipse. So a succession of ellipses can be used to characterize a trajectory. However, at each point more than one ellipse can be chosen since one has the freedom to choose in what direction the instantaneous ellipse is going through the point, i.e. what is the velocity at that point on that instantaneous ellipse. There are therefore three degrees of freedom when choosing to characterize a trajectory as a succession of ellipses. This flexibility is called gauge freedom.

Among all the possible ellipses one can choose from at each instant, there is a special choice whereby the velocity of the instantaneous ellipse is equal to the physical velocity $\mathbf{v}$ along the trajectory. That special ellipse is qualified as osculating. This description of the trajectory is explained in detail by Efroimsky [18].

If one uses equinoctial elements and assigns a function of $L$ to each of them to describe the evolution of the ellipses, then one can write the physical velocity as:

$$
\mathbf{v}=\frac{d \mathbf{r}}{d L} \frac{d L}{d t}=\frac{1}{t^{\prime}}\left(\frac{\partial \mathbf{r}}{\partial \mathbf{x}} \frac{d \mathbf{x}}{d L}+\frac{\partial \mathbf{r}}{\partial L}\right)
$$


Primes denote differentiations with respect to $L$. In order to obtain the osculating condition, one has to write down the velocity of the osculating orbit. The latter is obtained by differentiating (31) with respect to $L$, while assuming that all other elements are fixed. Therefore one has:

$$
\mathbf{v}_{o s c}=\frac{1}{t_{o s c}^{\prime}} \frac{\partial \mathbf{r}}{\partial L}
$$

$t_{o s c}^{\prime}=\dot{L}_{o s c}$ is the value obtained from the conservation of angular momentum and so:

$$
t_{o s c}^{\prime}=\frac{r^{2}}{\sqrt{\mu \hat{p}}}=\frac{1}{\sqrt{\mu \hat{p}}}\left(\frac{\hat{p}}{1+\hat{f} \cos L+\hat{g} \sin L}\right)^{2}
$$

The hats on the symbols denote the functions of $L$ that one sets to describe the evolution of the respective equinoctial elements. These functions shall be called shaping functions. Since the physical velocity can be written as the sum of an osculating term and a gauge term $\mathbf{v}_{\text {gauge }}$, one obtains the following expression for the latter:

$$
\begin{aligned}
\mathbf{v}_{\text {gauge }} & =\frac{1}{\hat{t}^{\prime}}\left(\frac{\partial \mathbf{r}}{\partial \mathbf{x}} \frac{d \mathbf{x}}{d L}+\frac{\partial \mathbf{r}}{\partial L}\right)-\frac{1}{t_{\text {osc }}^{\prime}} \frac{\partial \mathbf{r}}{\partial L} \\
& =\frac{1}{\hat{t}^{\prime}}\left[\left(1-\frac{\hat{t}^{\prime}}{t_{\text {osc }}^{\prime}}\right) \frac{\partial \mathbf{r}}{\partial L}+\frac{\partial \mathbf{r}}{\partial \mathbf{x}} \frac{d \mathbf{x}}{d L}\right] \\
& =\frac{1}{\hat{t}^{\prime}} \mathbf{\Phi}
\end{aligned}
$$

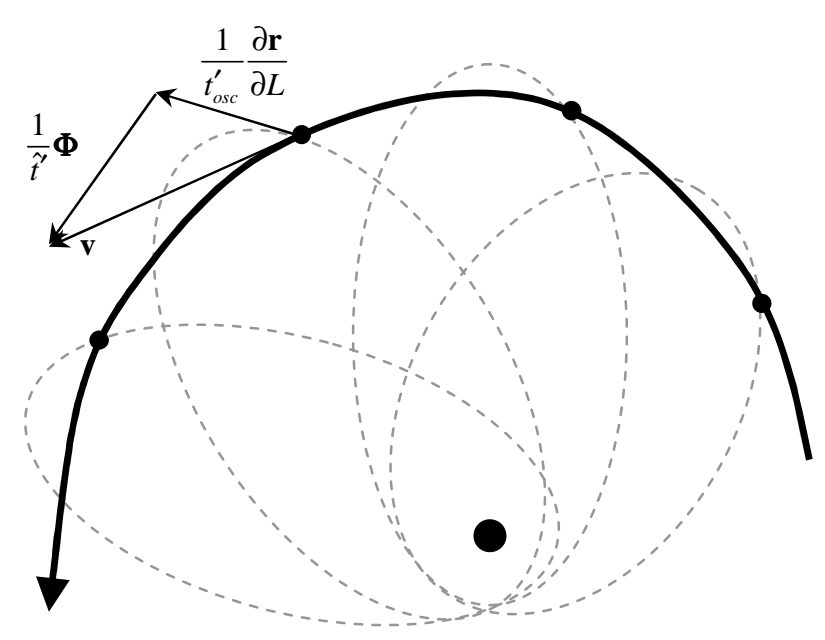

Fig. 3: Plot of a trajectory (bold line) whose points pass through instantaneous ellipses. In the illustrated case, the ellipses are not osculating because $\Phi \neq 0$.

Fig. 3 illustrates the decomposition of the velocity into $\mathbf{v}_{\text {osc }}$ and $\mathbf{v}_{\text {gauge }}$. The osculating condition imposes $\mathbf{v}_{\text {gauge }}=0$. Developing the latter results in a system of three nonlinear differential equations that define $\operatorname{Im}\left(f_{\mathbf{x}}\right)$. After simplification, they are written as: 


$$
\left\{\begin{array}{l}
\frac{\hat{p}^{\prime}}{r}-\hat{f}^{\prime} \cos L-\hat{g}^{\prime} \sin L=\left(\hat{t}^{\prime} \frac{\sqrt{\mu \hat{p}}}{r^{2}}-1\right)(\hat{f} \sin L-\hat{g} \cos L) \\
\frac{\hat{h} \hat{k}^{\prime}-\hat{k}^{\prime}}{1+\hat{h}^{2}+\hat{k}^{2}}=1-\hat{t}^{\prime} \frac{\sqrt{\mu \hat{p}}}{r^{2}} \\
\hat{h}^{\prime} \sin L-\hat{k}^{\prime} \cos L=0
\end{array}\right.
$$

It is generally accepted to call the set of elements described by $\hat{p}, \hat{f}, \hat{g}, \hat{h}, \hat{k}$ and $L$ equinoctial only when they describe osculating orbits, and pseudo-equinoctial otherwise. The Gauss planetary equations are obtained from the three equations of motion and the three osculating conditions. Therefore if the parameters defining $\mathbf{x}$ were computed from the propagation of Gauss planetary equations, under a low-thrust action, then they would be classical osculating nonsingular equinoctial elements.

In the pseudo-equinoctial shaping, however, the dependency of all five elements $p, f, g, h$ and $k$ on $L$ is defined by arbitrary shaping functions. In particular, the following functions were proposed in [11]:

$$
\left\{\begin{array}{l}
\hat{p}(L)=p_{0}+p_{1} \exp \lambda_{1} L \\
\hat{f}(L)=f_{0}+f_{1} \exp \lambda_{2} L \\
\hat{g}(L)=g_{0}+g_{1} \exp \lambda_{2} L \\
\hat{h}(L)=h_{0}+h_{1} \exp \lambda_{3} L \\
\hat{k}(L)=k_{0}+k_{1} \exp \lambda_{3} L
\end{array}\right.
$$

The parameters $\lambda_{1}, \lambda_{2}, \lambda_{3}$ are called shaping parameters and they are additional degrees of freedom that one can use to modify the shape of the trajectory.

The shaping function for the time component of the state vector, as in the spherical shaping method, is defined through its derivative with respect to the parameter $L$, for which the following expression was used in the present study:

$$
\hat{t}^{\prime}=t_{o s c}^{\prime}=\frac{1}{\sqrt{\mu \hat{p}}}\left(\frac{\hat{p}}{1+\hat{f} \cos L+\hat{g} \sin L}\right)^{2}
$$

Adopting the latter expression is like assuming that the out-of-plane component of the control vector is negligible with respect to the magnitude of the gravity field. The gauge function has then the expression $\mathbf{\Phi}=\frac{\partial \mathbf{r}}{\partial \mathbf{x}} \frac{d \mathbf{x}}{d L}$. 
It can be verified that the shaping of the elements in Eqs. (37) and (38) does not satisfy $\boldsymbol{\Phi}=0$ and therefore these elements do not represent an osculating orbit. Hence, they represent an orbit which passes through the same point as the osculating one but with a different velocity. It can be shown indeed that if (38) is chosen for the expression of $\hat{t}^{\prime}$, then osculating conditions would impose $\hat{h}^{\prime}=\hat{k}^{\prime}=0$, i.e. the transfer is planar, and

$$
\frac{\hat{p}^{\prime}}{\hat{p}}(1+\hat{f} \cos L+\hat{g} \sin L)-\hat{f}^{\prime} \cos L-\hat{g}^{\prime} \sin L=0
$$

What actually happens is that by imposing a shape for the elements in $\mathbf{x}$ one fixes the gauge function $\boldsymbol{\Phi}$. If that function is not zero, i.e. the Lagrange constraint is not satisfied, then the elements in $\mathbf{x}$ are not osculating and do not satisfy the Gauss planetary equations.

Providing $\hat{t}$ from (38) and arbitrary shaping functions $\hat{p}, \hat{f}, \hat{g}, \hat{h}, \hat{k}$ defines the position through Eq. (31), the gauge function $\boldsymbol{\Phi}$ and the velocity through $\mathbf{v}=\left(\frac{\partial \mathbf{r}}{\partial L}+\boldsymbol{\Phi}\right) \frac{1}{\hat{t}^{\prime}}$. Reciprocally, it can be shown that if one provides the position $\mathbf{r}$, the velocity $\mathbf{v}$ and an arbitrary gauge function $\boldsymbol{\Phi}$, and assumes that $\hat{t}$ is defined as in (38), then the corresponding profiles for $\hat{p}, \hat{f}, \hat{g}, \hat{h}$ and $\hat{k}$ exist and are unique. In fact, $\hat{p}, \hat{f}, \hat{g}, \hat{h}, \hat{k}$ and $L$ are the osculating equinoctial elements corresponding to the position $\mathbf{r}$ and the velocity $\mathbf{v}-\boldsymbol{\Phi} \frac{\|\mathbf{v}\|}{\left\|\frac{\partial \mathbf{r}}{\partial L}+\boldsymbol{\Phi}\right\|}$. The special case of $\frac{\partial \mathbf{r}}{\partial L}+\boldsymbol{\Phi}=\mathbf{0}$ corresponds to $\mathbf{v}=0$, which is rarely encountered in practice. It is worth noting that the component $t$ of $\mathbf{x}$ is absent in the expression of $\boldsymbol{\Phi}$ because $\frac{\partial \mathbf{r}}{\partial t}=0$.

The coefficients $p_{0}, f_{0}, g_{0}, h_{0}, k_{0}$ and $p_{1}, f_{1}, g_{1}, h_{1}, k_{1}$ are computed by satisfying the boundary conditions on position and velocity. A Newton loop is performed to satisfy the boundary conditions exactly, since the sextuplet of functions $\left(\begin{array}{llllll}\hat{p} & \hat{f} & \hat{g} & \hat{h} & \hat{k} & L\end{array}\right)$ does not define osculating equinoctial elements. The Newton loop is initialized with values for the coefficients that provide the osculating values for $\left(\begin{array}{llllll}\hat{p} & \begin{array}{l}\hat{f} \\ \hat{g}\end{array} \hat{h} & \hat{k} & \hat{t}\end{array}\right)$ at the boundaries. In mathematical terms, the osculating values correspond to the solution to: 


$$
\left\{\begin{array}{l}
\mathbf{r}\left(\mathbf{x}_{i}, L_{i}\right)=\mathbf{r}_{i} \\
\left.\frac{1}{t_{o s c}^{\prime}\left(\mathbf{x}_{i}, L_{i}\right)} \frac{\partial \mathbf{r}}{\partial L}\right|_{\left(\mathbf{x}_{i}, L_{i}\right)}=\mathbf{v}_{i} \\
\mathbf{r}\left(\mathbf{x}_{f}, L_{f}\right)=\mathbf{r}_{f} \\
\left.\frac{1}{t_{o s c}^{\prime}\left(\mathbf{x}_{f}, L_{f}\right)} \frac{\partial \mathbf{r}}{\partial L}\right|_{\left(\mathbf{x}_{f}, L_{f}\right)}=\mathbf{v}_{f}
\end{array}\right.
$$

From $\left(\mathbf{x}_{i}, L_{i}\right)$ and $\left(\mathbf{x}_{f}, L_{f}\right)$ one gets the values of the coefficients by solving the linear system (37). These coefficients are used to initialize the Newton loop to satisfy the boundary constraints. If the coefficients inside the shaping functions are assembled into a vector $\mathbf{c}$ with 10 components, then the solution to the following system in $\left(\mathbf{c}, L_{i}, L_{f}\right)$ is searched for iteratively:

$$
\left\{\begin{array}{l}
\mathbf{r}\left(\mathbf{x}\left(\mathbf{c}, L_{i}\right), L_{i}\right)=\mathbf{r}_{i} \\
\frac{1}{\hat{t}^{\prime}\left(\mathbf{x}\left(\mathbf{c}, L_{i}\right), L_{i}\right)}\left[\left.\frac{\partial \mathbf{r}}{\partial L}\right|_{\left(\mathbf{x}\left(\mathbf{c}, L_{i}\right), L_{i}\right)}+\boldsymbol{\Phi}\left(\mathbf{x}\left(\mathbf{c}, L_{i}\right), L_{i}\right)\right]=\mathbf{v}_{i} \\
\mathbf{r}\left(\mathbf{x}\left(\mathbf{c}, L_{f}\right), L_{f}\right)=\mathbf{r}_{f} \\
\frac{1}{\hat{t}^{\prime}\left(\mathbf{x}\left(\mathbf{c}, L_{f}\right), L_{f}\right)}\left[\left.\frac{\partial \mathbf{r}}{\partial L}\right|_{\left(\mathbf{x}\left(\mathbf{c}, L_{f}\right), L_{f}\right)}+\boldsymbol{\Phi}\left(\mathbf{x}\left(\mathbf{c}, L_{f}\right), L_{f}\right)\right]=\mathbf{v}_{f}
\end{array}\right.
$$

Eqs. (41) are a system of 12 equations with 12 unknowns. Using the coefficients from the osculating elements can be expected to be a good starting point for solving the system as long as the gauge function remains small, i.e. the pseudo-equinoctial elements are not too far from being osculating. In mathematical terms, this condition translates into:

$$
\|\boldsymbol{\Phi}\| \ll\left\|\frac{\partial \mathbf{r}}{\partial L}\right\|
$$

Due to the fact that $\boldsymbol{\Phi}=\frac{\partial \mathbf{r}}{\partial \mathbf{x}} \frac{d \mathbf{x}}{d L}$ and that one can expect the orders of magnitude of each $\frac{\partial \mathbf{r}}{\partial x_{j}}$ be the same as the one of $\frac{\partial \mathbf{r}}{\partial L}$, the condition (42) becomes ultimately $\left|\frac{d x_{i}}{d L}\right| \ll\left|x_{i}\right|$. The meaning of the latter is that the shaping functions should not have too abrupt variations. Therefore, no matter which expressions for the shaping functions are used, the 
shorter the transfer in terms of $L_{f}-L_{i}$, the farther the osculating initial guess will be from the solution of (41) and the smaller will be the chance that the Newton loop converges.

Once the values of the longitudinal anomalies at the boundaries and the coefficients inside the shaping functions of the pseudo-equinoctial elements are obtained, the trajectory is completely characterized. $\hat{t}^{\prime}$ is defined through Eq. (38) and the evolution of time is then computed by solving the integral:

$$
t=\hat{t}(L)=\int_{L_{i}}^{L} \hat{t}^{\prime}(l) d l
$$

The time of flight is uniquely defined. If the time of flight is constrained, then a second Newton loop over one of the shaping parameters can be used to satisfy that constraint. Finally, the total $\Delta v$ is obtained by integrating $|\mathbf{u}| \hat{t}^{\prime}$ over $\left[L_{i} L_{f}+2 n_{r} \pi\right]$. The magnitude of the $\Delta v$ can vary substantially, depending on the time of flight to be satisfied.

\section{Satisfaction of the time of flight constraint}

The advantage of generating trajectories by shaping the state vectors is that the equations of motion provide the corresponding control law analytically. However there are physical quantities which are more difficult to obtain with this method, one of them is the time of flight corresponding to a given shape. The time of flight is defined as $T\left(s_{f}\right)-T\left(s_{i}\right)$ and is a function of the parameter $s$. If the derivative of $T$ is provided, as in the case of the shaping methods described above, then the time of flight is the integral of $T^{\prime}$ over $\left[\begin{array}{ll}s_{i} & s_{f}\end{array}\right]$. The analytical integral of $T^{\prime}$ is not generally possible and has to be solved numerically.

The time of flight is often constrained as the spacecraft has to arrive at destination at a given time to rendezvous or fly by a celestial body. In order to satisfy that constraint, at least one additional parameter is required when shaping the trajectory. A way to satisfy automatically the time of flight constraint is by shaping $T$ or $T$ ' such that the desired time of flight $T_{f}$ is exactly $T\left(s_{f}\right)-T\left(s_{i}\right)=\int_{s_{i}}^{s_{f}} T^{\prime} d s$. One would be tempted to choose an expression for $T^{\prime}$ that can be analytically reduced to quadrature. Although this approach would be computationally the ideal way to solve the time of flight constraints, the resulting thrust profiles would not necessarily have reasonable magnitudes in practice. That explains why, in the previous subsections, the time evolution profiles were chosen to correspond either to a tangential thrust or a $2 \mathrm{D}$ motion.

If the parameterization is performed using the time $t$, one could then trivially define the trajectory in the desired segment $\left[\begin{array}{ll}t_{i} & t_{f}\end{array}\right]$. This approach would remove the need to address any time of flight constraints further down in the calculations. On the other hand, quantities like the azimuthal angle $\theta$ or the longitudinal anomaly $L$ would need to be 
constrained to take values within the desired limits such that the boundary conditions are satisfied. The problem of satisfying the time of flight constraint is then replaced by the problem of satisfying the boundary conditions.

A two step approach to address the time of flight constraints is presented in this subsection, the second step being applied if the first one fails. The two steps differ in the way the additional parameter is used in the formulation of the shaping. The first one includes the parameter within the expression of the functions shaping the state vector. The second approach consists of augmenting the initial time evolution function $T$ in a way that the time of flight constraint is exactly satisfied.

\section{Inserting an additional parameter within the shaping functions of the state vector}

This approach can be applied to all the shaping methods described in this paper. It consists of adding a degree of freedom to the expression of one of the functions shaping the state vectors. The idea is that when one varies the value of the additional parameter, the time of flight varies, and the problem translates into the search for the right value of the parameter that satisfies the time constraint. Due to the nonlinear relationship between the time of flight and the shaping parameters, it is generally impossible to solve the problem analytically. Here, the Newton iteration for the solution of nonlinear equations was used.

\section{Augmenting the original time of flight evolution}

If the time $t$ is decoupled from the other state variables in Eq.(5), instead of inserting the additional parameter within the expression of the shaping function, one can insert the additional parameter in the definition of $t=T(s)$.

Let us suppose that an initial trajectory, provided by a shaping method, has a time profile $t=T(s)$. Without loss of generality, one can take $T\left(s_{i}\right)=0$ and $T\left(s_{f}\right)$ equal to the computed time of flight. If $T_{f}$ is the desired time of flight and $T_{\text {viol }}=T\left(s_{f}\right)-T_{f}$ is the time of flight violation, one can introduce a function $\chi$ satisfying $\chi\left(s_{f}\right)-\chi\left(s_{i}\right)=1$ such that the time profile is $T_{\chi}=T-T_{\text {viol }} \chi$, with $T$ that verifies $T_{\chi}\left(s_{f}\right)=T_{f}$. The shaping of the time must be such that $T_{\chi}$ is strictly monotonous and $T_{\chi}^{\prime}$ never becomes 0 , otherwise singularities occur when calculating the control law. The simplest form that $\chi$ can take is $\chi(s)=\left(s-s_{i}\right) / \Delta s$ where $\Delta s=s_{f}-s_{i}$, however it is often the case that boundary conditions exist on $\dot{s}$ and thus on $T_{\chi}^{\prime}$. Therefore, the function $\chi$ must satisfy three conditions:

$$
\begin{aligned}
& \chi^{\prime}\left(s_{i}\right)=0 ; \\
& \chi^{\prime}\left(s_{f}\right)=0 ; \\
& \chi\left(s_{f}\right)-\chi\left(s_{i}\right)=1
\end{aligned}
$$


The last condition can be rewritten as $\int \chi^{\prime} d s=1$. Eqs. (44) can be satisfied by choosing a polynomial of degree two for $\chi^{\prime}$ such as $\chi^{\prime}(s)=-6\left(s-s_{i}\right)\left(s-s_{f}\right) / \Delta s$.

This method for satisfying the time of flight constraints is faster than the use of the Newton loop since only two iterations are needed to find the desired trajectory: the first iteration computes the time of flight violation $T_{v i o l}$, the second recalculates the dynamics with $T_{\chi}=T-T_{\text {viol }} \chi$.

It should be noted, however, that if this method was applied alone to satisfy the time of flight constraints, then undesired phenomena might occur. In fact, the addition of $-T_{\text {viol }} \chi$ to the time evolution profile can distort the initial low-thrust character of the dynamics, and the resulting control profile can have a high magnitude. The method can also break down in some cases when $T_{\chi}=T-T_{\text {viol }} \chi$ stops being strictly monotonous, in which case singularities occur when calculating $\dot{s}=1 / t^{\prime}$. These inconvenient behaviors led to the decision to use this method only when the Newton loop fails to satisfy the time of flight constraint.

\section{E. Test cases}

Three mission scenarios were selected to test the shaping methods and the method to satisfy the time of flight constraint: a rendezvous mission from the Earth to Mars, to the near Earth asteroid 1989ML, to comet Tempel-1 and to Neptune. The orbital elements of the four target bodies are listed in Table 1.

Systematic searches were conducted on a wide range of launch windows and times of flight, in order to evaluate the overall capacity of the shaping methods to model low thrust transfers. The characteristics of the spacecraft and its dynamics are the same in all three cases. The spacecraft is represented by a point with a mass of $1000 \mathrm{~kg}$. It carries a propulsion system with a specific impulse of $3000 \mathrm{~s}$. No limit on the achievable thrust magnitude is assumed. The spacecraft is subject only to the gravitational pull of a central body (the Sun in all three cases) and to the propulsion system.

The shapes were implemented in a Matlab code with all computations performed on an Intel Core 2 Duo processor running Linux.

Table 1 Orbital elements of Mars, near Earth asteroid 1989ML and Tempel-1

\begin{tabular}{lcccc}
\hline \hline & Mars & $1989 \mathrm{ML}$ & Tempel-1 & Neptune \\
\hline Semi-major axis & $1.524 \mathrm{AU}$ & $1.272 \mathrm{AU}$ & $3.124 \mathrm{AU}$ & $30.104 \mathrm{AU}$ \\
Eccentricity & 0.093 & 0.137 & 0.517 & 0.011 \\
Inclination & $1.850^{\circ}$ & $4.378^{\circ}$ & $10.527^{\circ}$ & $1.768^{\circ}$ \\
Right ascension & $49.557^{\circ}$ & $104.411^{\circ}$ & $68.933^{\circ}$ & $131.794^{\circ}$ \\
Argument of periapsis & $286.502^{\circ}$ & $183.267^{\circ}$ & $178.926^{\circ}$ & $265.647^{\circ}$ \\
\hline \hline
\end{tabular}


The best shaped solutions were then used as initial guess for the direct trajectory analysis tool DITAN. DITAN takes the control profile resulting from the shaping as input and transcribes the optimal control problem associated to low-thrust trajectories with finite elements in time generated on spectral bases [11]. In this subsection, DITAN was run taking as maximum thrust level the peak thrust obtained from the shaping approaches, and minimizing the propellant mass, with the aim of assessing how close to optimal the $\Delta v$ s provided by the shaping methods are.

\section{Rendezvous with Mars}

The launch date $t_{\mathrm{i}}$ considered for this mission covers the period between January $1^{\text {st }} 2020$ and December $31^{\text {st }} 2027$ and is discretized with a 15-day time step. This window is large enough to contain almost four synodic periods of Mars (2.14 years). The time of flight ranges between 500 and 2000 days and is discretized with a 20-day time step size. The number of revolutions $n_{\mathrm{r}}$ allowed for the transfers is between 1 and 4 .

Table 2 Results of each shaping method for the Mars rendezvous mission.

\begin{tabular}{lll}
\hline \hline & Spherical & Pseudo-equinoctial \\
\hline Percentage of feasible trajectories & $100 \%$ & $89.1 \%$ \\
$\Delta v$ of the best trajectory $[\mathrm{km} / \mathrm{s}]$ & 5.74 & 5.83 \\
Peak thrust of the shaped trajectory with the best $\Delta v[\mathrm{~N}]$ & 0.22 & 0.16 \\
DITAN optimized $\Delta \mathrm{v}[\mathrm{km} / \mathrm{s}]$ & 5.69 & 5.68 \\
Average computational time for shaping a trajectory $[\mathrm{s}]$ & 0.316 & 0.238 \\
\hline \hline
\end{tabular}

Trajectories were deemed feasible if the time of flight constraints were satisfied. Table 2 shows the percentage of feasible trajectories obtained through the systematic search for both the spherical and pseudo-equinoctial shaping methods. The $\Delta v$ of the best solution from each shaping is also presented together with the corresponding optimal solution when fed into DITAN. Note that the trajectories with the lowest $\Delta v$ provided by the spherical shaping and by the pseudo-equinoctial shaping are different. A limit on the thrust was set when performing the optimization, equal to the peak thrust of the shaped trajectory. Table 2 also reports the average time required to generate a solution with the shaping approach. Fig. 4 represents all the feasible solutions with their associated $\Delta v$ cost. Note how the spherical shape provides a wider set of feasible solution with lower $\Delta v$. On the other hand both shapes identify the same regions in the $t_{\mathrm{i}}-T_{f}$ space where the transfer requires a high $\Delta v$. These regions are located towards the lower values of $T_{f}$. A periodic pattern can be observed in the plots, where the period corresponds to the Earth-Mars synodic period. 


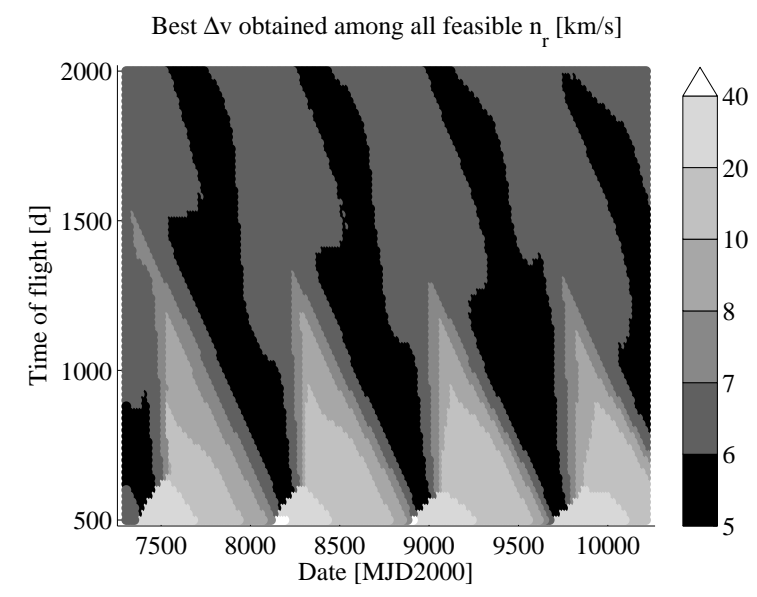

(a)

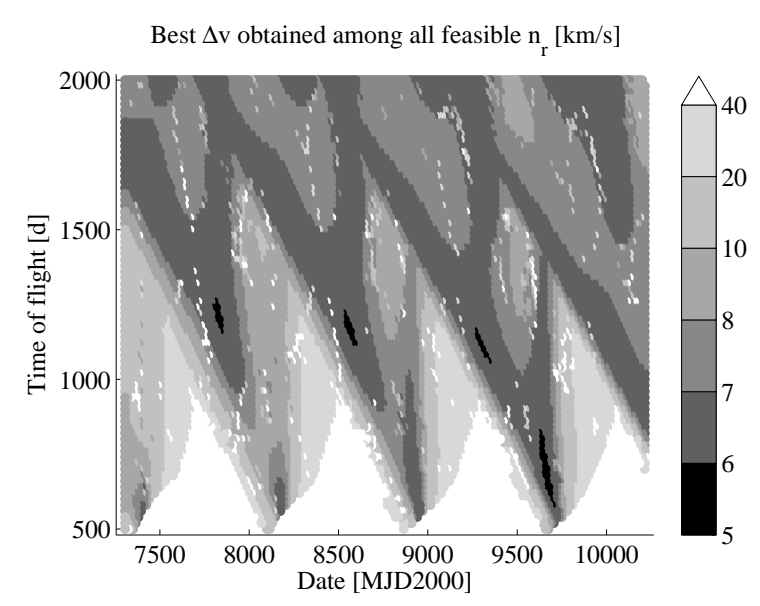

(b)

Fig. 4 Illustration of the set of combinations of launch date and time of flight for which the spherical (a) and the pseudo-equinoctial shaping (b) found feasible solutions to rendezvous Mars.

\section{Rendezvous with Near Earth Asteroid 1989ML}

The launch window is the same as for the Mars case and was discretized with the same time step. The synodic period of asteroid 1989ML is 3.30 years, thus the launch window includes two full synodic periods. The range of the time of flight is between 100 and 1000 days, and is discretized with a 20-day time step size. The number of revolutions $n_{\mathrm{r}}$ allowed for the transfers is between 1 and 2 .

Table 3 Results of each shaping method for the 1989ML rendezvous mission.

\begin{tabular}{lll}
\hline \hline & Spherical & Pseudo-equinoctial \\
Percentage of feasible trajectories & $83.7 \%$ & $75.5 \%$ \\
$\Delta v$ of the best trajectory [km/s] & 4.47 & 4.82 \\
Peak thrust of the shaped trajectory with the best $\Delta v[\mathrm{~N}]$ & 0.31 & 0.33 \\
DITAN optimized $\Delta v[\mathrm{~km} / \mathrm{s}]$ & 4.21 & 4.45 \\
Average computational time for shaping a trajectory $[\mathrm{s}]$ & 0.316 & 0.264 \\
\hline \hline
\end{tabular}

Table 3 presents the percentage of feasible solutions for both the spherical and pseudo-equinoctial shaping. The behavior of the shaping method is similar to the case of the Mars rendezvous mission (see Fig. 4). The $\Delta v$ 's are on average lower than for the Mars mission, because the semi-major axis of 1989ML is lower. The difference in orbital inclination between Mars and 1989ML is only 2.5 degrees and it has a weaker impact on the total $\Delta v$ than a difference of semi-major axis of 0.25 AU. The percentage of feasible trajectories is lower than for the Mars mission because the second step of the method to satisfy the time of flight results in a singularity, due to the behavior explained in Section II.D. Indeed, the Newton loop for satisfying the time of flight does not converge for the cases where the desired time of flight is very low compared to the number of revolutions. The reshaping of the time 
evolution $T$ takes over in that case. However, the value of $T_{\text {viol }}$ is too high causing $T^{\prime}$ to tend towards 0 , and the resulting trajectory is not physical. Fig. 5 is a plot of the departure dates and times of flight of the feasible trajectories for both shaping methods. It can be observed that the spherical shaping always provides at least one feasible trajectory if the time of flight is above 300 days. For numbers of revolution that are unreasonable compared to the desired time of flight, both shaping methods break down. The pseudo-equinoctial shaping provides fewer feasible trajectories than the spherical shaping, because the shaping parameters do not give enough flexibility to change the shapes and attain wide ranges of times of flight. However similar patterns can be observed in the results of both shaping methods, which hints to better suited relative configurations between Earth and 1989ML for a lowthrust transfer. The patterns show a periodicity equal to the value of the synodic period of the Earth-1989ML system.

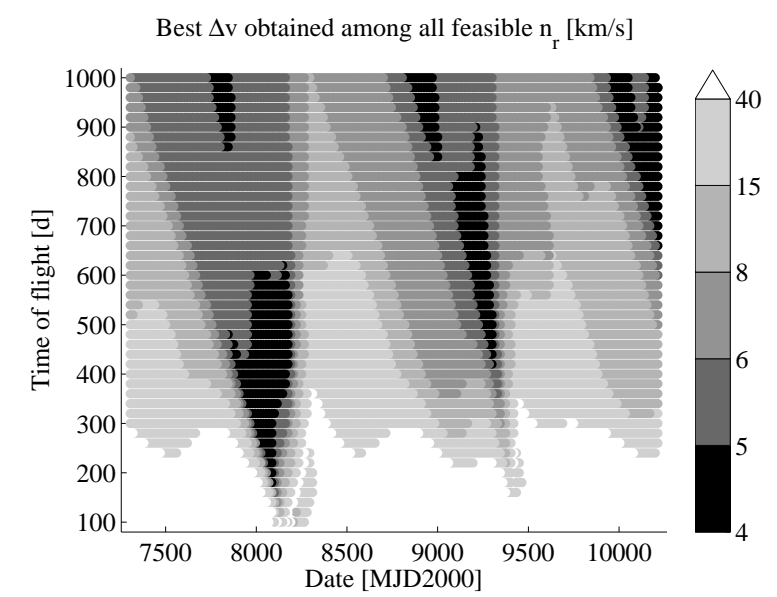

(a)

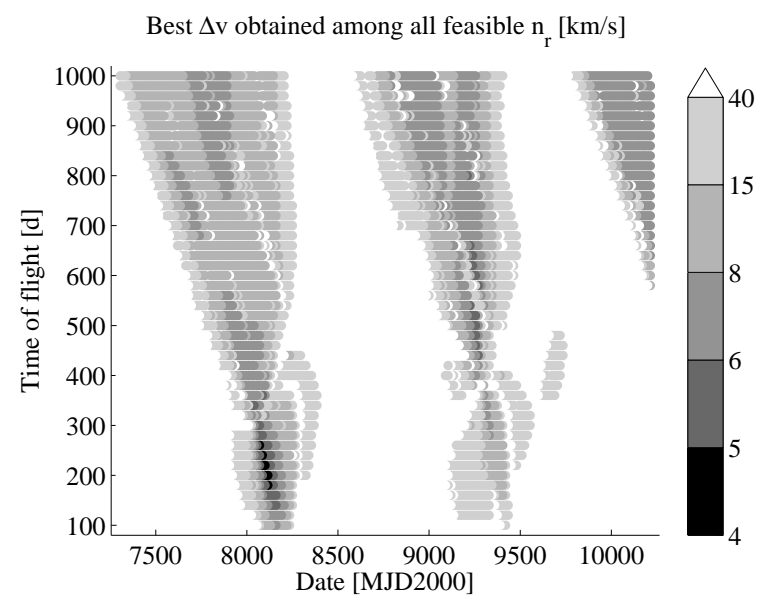

(b)

Fig. 5 Illustration of the set of combinations of launch date and time of flight for which the spherical (a) and the pseudo-equinoctial (b) shaping method found feasible solutions to rendezvous 1989ML.

\section{Rendezvous with comet Tempel-1}

Tempel-1 was chosen as a target because it has a very eccentric and relatively inclined orbit. McConaghy et al. [21] used this test case for the exponential sinusoid shaping. A systematic search was performed on the same launch window proposed by McConaghy et al.: between January $1^{\text {st }} 2000$ and January $3^{\text {rd }} 2016$. The range of the time of flight is between 400 and 1500 days, and the number of revolutions $n_{\mathrm{r}}$ was set between 0 and 2 .

Table 4 Results of each shaping method for the Tempel-1 rendezvous mission.

\begin{tabular}{lcc}
\hline \hline & Spherical & $\begin{array}{c}\text { Pseudo- } \\
\text { equinoctial }\end{array}$ \\
\hline $\begin{array}{l}\text { Percentage of feasible trajectories } \\
\Delta v \text { of the best trajectory }[\mathrm{km} / \mathrm{s}]\end{array}$ & $68.1 \%$ & $43.2 \%$ \\
& 11.13 & 13.44
\end{tabular}


Peak thrust of the shaped trajectory with the best $\Delta v[\mathrm{~N}]$

Table 4 presents the percentage of feasible solutions for both the spherical and pseudo-equinoctial shaping. The behavior of the two shaping methods is similar to the previous two cases; however fewer trajectories are feasible because the Newton loop fails to converge more often, although for every launch date at least one feasible trajectory exists. McConaghy et al. present the propellant mass fractions resulting from the exponential sinusoids. A constant specific impulse of 3000 seconds is used to convert the low-thrust $\Delta v$ from the exponential sinusoid. Using this value for the specific impulse, the $\Delta v$ of $11.13 \mathrm{~km} / \mathrm{s}$ of the best transfer from the spherical shaping converts into a propellant mass fraction of $31.5 \%$. The pseudo-equinoctial's best $\Delta v$ of $13.44 \mathrm{~km} / \mathrm{s}$ converts into $36.7 \%$ of propellant mass fraction. No impulsive $\Delta v$ s are to be taken into account because the boundary constraints on velocity are satisfied. A substantial improvement is obtained compared to McConaghy et al. whose best shaped trajectory requires $50 \%$ of propellant mass fraction.

Fig. 6 illustrates the set of feasible combinations of launch dates and times of flight found by the spherical and the pseudo-equinoctial shaping methods. When more than one number of revolutions is feasible for a given combination of launch date and time of flight then only the one with the lowest $\Delta v$ is plotted. Both shaping methods identify the same region where the transfer is too costly in $\Delta v$. These regions are periodically distributed, with the period of Tempel 1. A smaller scale periodicity also exists, and corresponds to the Earth's period (and close to the synodic period of the Earth-Tempel 1 system). It can be therefore deduced from the plots that the arrival position on Tempel 1's orbit has more impact than the departure position on Earth's orbit. There is physical sense in this observation because the transfer can change substantially if the spacecraft arrives at Tempel 1's perihelion at 1.51 AU or apohelion at 4.74 AU. Indeed, in the first case the perihelion is raised first, followed by the apohelion just before arrival, and in the second case the order of the two maneuvers is inversed. Finally, the results show that the transfers are generally more costly in $\Delta v$ when the time of flight shortens. 


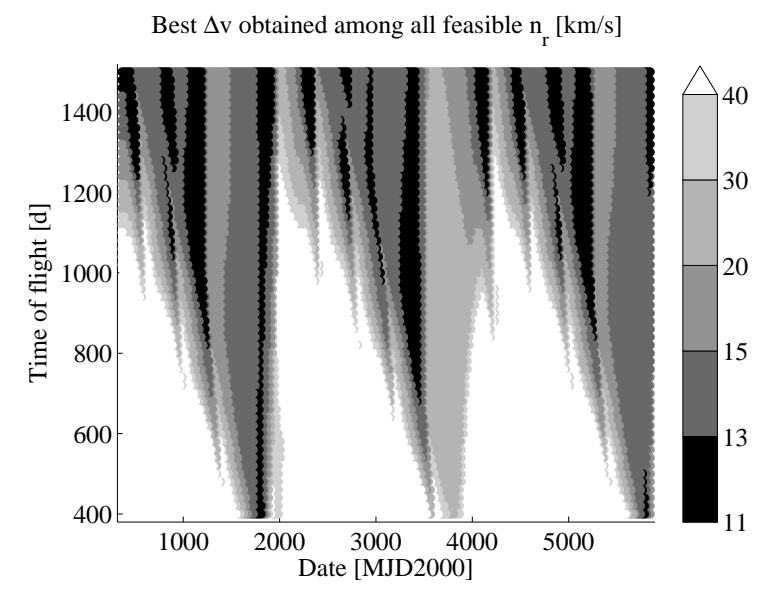

(a)

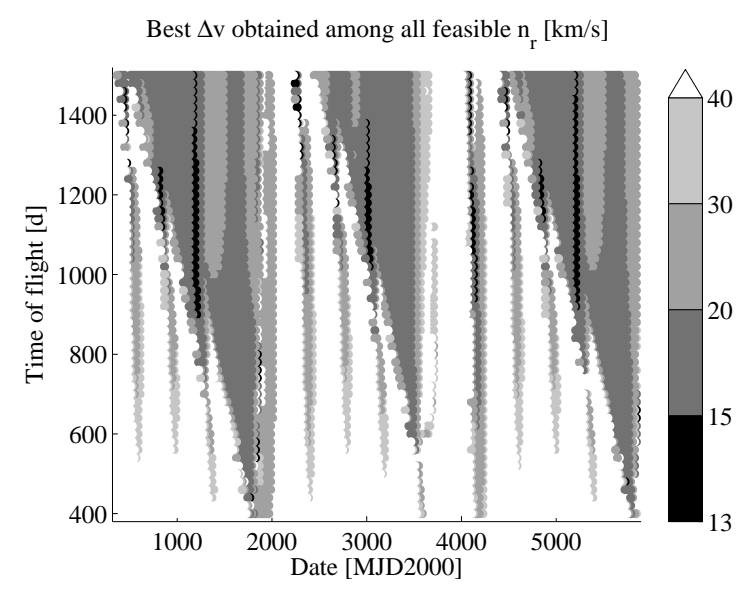

(b)

Fig. 6 Illustration of the set of combinations of launch date and time of flight for which the spherical (a) and the pseudo-equinoctial (b) shaping method found feasible solutions to rendezvous Tempel 1.

\section{Rendezvous with Neptune}

In order to test the shaping methods on a wide range of transfer types, a rendezvous with Neptune is also studied as test case. Neptune has a semi-major axis of $30.1 \mathrm{AU}$ and an orbital period of 164.8 years. One can make initial estimations of the orders of magnitude involved in a rendezvous to Mars by studying the Hohmann transfer between two circular orbits representing Earth's and Neptune's. Straightforward computations provide the characteristics of the Hohmann transfer. The transfer ellipse has a semimajor axis of $15.6 \mathrm{AU}$ and eccentricity of 0.94 and the transfer time is 30.7 years. The first maneuver at Earth has a $\Delta v$ of $11.66 \mathrm{~km} / \mathrm{s}$ and the second one at Neptune is of 4.05 $\mathrm{km} / \mathrm{s}$, so the total Hohmann transfer requires a $\Delta v$ of $15.71 \mathrm{~km} / \mathrm{s}$.

A systematic search was performed over a launch window between January $1^{\text {st }} 2020$ and December $31^{\text {st }} 2025$, discretized at every 15 days. Two scenarios have been addressed: one without heliocentric revolutions and one with 10 revolutions. The values of times of flight that were investigated differed between the two cases. For no revolutions that set ranged between 11000 and 30000 days (that is 30.1 and 82.1 years) at 500 day time steps. For the case of 10 revolutions, the times of flight ranged between 40000 and 80000 days, with intervals of 500 days. An initial tangential velocity of $3 \mathrm{~km} / \mathrm{s}$ relative to Earth was set at the departure for the transfers without revolutions, while in the other case the initial relative velocity at the Earth is zero.

Table 5 Results of each shaping method for the Neptune rendezvous mission with no revolution

\begin{tabular}{lcc}
\hline \hline & Spherical & $\begin{array}{c}\text { Pseudo- } \\
\text { equinoctial }\end{array}$ \\
\hline Percentage of feasible trajectories & $92.4 \%$ & $13.6 \%$ \\
$\Delta v$ of the best trajectory $[\mathrm{km} / \mathrm{s}]$ & 14.99 & 50.37
\end{tabular}



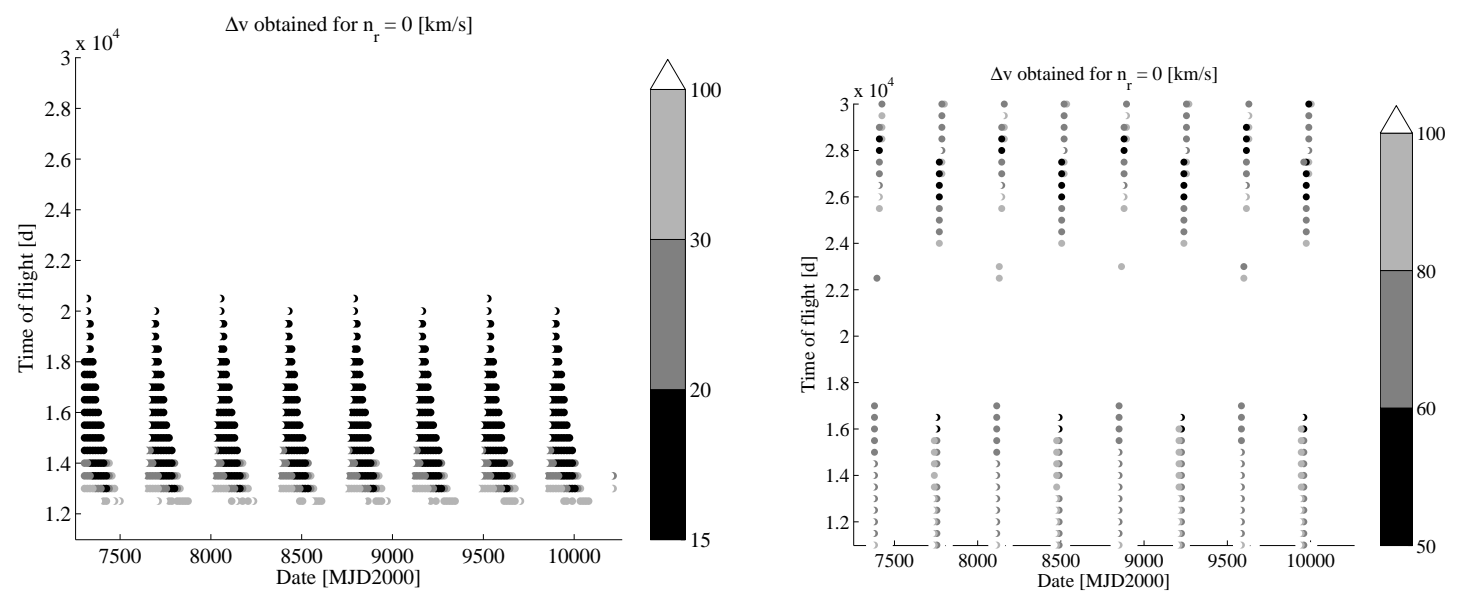

Fig. 7 Illustration of the set of combinations of launch date and time of flight for which the spherical (a) and the pseudo-equinoctial (b) shaping method found feasible solutions to rendezvous Neptune, without any revolution.

It can be seen from Fig. 7 that the two shaping methods provide different results when no revolutions are allowed. The spherical shaping produces transfers with substantially lower $\Delta v$ s, with the lowest values of $\Delta v$ reaching $15 \mathrm{~km} / \mathrm{s}$. The results have a periodicity of a year, which is the synodical period of the Sun-Earth-Neptune system. With the spherical shaping, the lowest $\Delta v$ s are obtained for transfers between 13000 days and 20000 days. Shaping the pseudo-equinoctial elements does not provide interesting results for two reasons. The first is that the Newton loop does not impose the boundary conditions well, the other is that the range of times of flight covered by varying the shaping parameter $\lambda_{l}$ is limited and the reshaping of the time evolution has to be used, which can potentially raise the $\Delta v$ s by much.

\section{Discussion}

Both shaping methods generated a number of feasible solutions for every launch date, although the Newton loop failed to converge for a number of times of flight. In particular, the loop did not converge when the flight time is short for the given number of revolutions. The cases in which the Newton loop fails correspond to trajectories with very high $\Delta v$ 's and as such, are often not interesting in practice. It should be noted, however, that due to the imposed shape there is no guarantee that the thrust magnitude is close to the optimal one. More importantly, the peak thrust recovered from the dynamic equations might be higher than the maximum thrust allowed for the transfer. This 
problem will be addressed in the remainder of this paper and represents a limitation of the shaping approach as it does not allow for a clear discrimination of the feasibility of a transfer given a specific engine.

\section{Linear quadratic controller}

This section describes a method to quickly improve the quality of the shaped solutions. The assumption behind this approach is that if the shaped solution is not locally optimal then there exists an optimal solution in a neighborhood of the shaped one. The validity of this assumption will be verified theoretically at the end of this section.

\section{A. Derivation of the LQ controller}

Let one assume that a spacecraft has position $\mathbf{r}$, velocity $\mathbf{v}$ and is subject to the gravitational pull of a central body with a gravity parameter $\mu$.. Additionally the spacecraft has an onboard controllable propulsion system that contributes to the motion of the spacecraft with an acceleration $\mathbf{u}$. If one defines the state vector $\mathbf{x}$ as $\mathbf{x}=[\mathbf{r}, \mathbf{v}]^{T}$ then the equations of motion can be written as $\dot{\mathbf{x}}=\mathbf{A}(\mathbf{x})+\mathbf{B u}$, with $\mathbf{A}(\mathbf{x})=\left[\mathbf{0},-\mu \mathbf{r}^{T} / r^{3}\right]^{T}$. The equations of motion are then linearized in the neighborhood of the nominal $\mathbf{x}_{\mathbf{0}}$ and $\mathbf{u}_{0}$ within the time interval $I_{t}$. The linearized system is:

$$
\left\{\begin{array}{l}
\xi\left(t_{i}\right)=[0,0,0,0,0,0]^{T} \\
\dot{\xi}=\left.\nabla \mathbf{A}\right|_{\mathbf{x}_{0}(t)} \boldsymbol{\xi}+\mathbf{B} \boldsymbol{v}=\left.\nabla \mathbf{A}\right|_{\mathbf{x}_{0}(t)} \boldsymbol{\xi}+\mathbf{B}\left(\mathbf{u}_{l}-\mathbf{u}_{0}(t)\right)
\end{array}\right.
$$

The gradient of $\mathbf{A}$ at a point $\mathbf{x}$ is expressed as:

$$
\nabla \mathbf{A}=\left[\begin{array}{ll}
\mathbf{O}_{3} & \mathbf{I}_{3} \\
\mathbf{A}_{g} & \mathbf{O}_{3}
\end{array}\right]
$$

such that $\mathbf{O}_{3}$ is the nil square matrix of order $3, \mathbf{I}_{3}$ is the identity matrix of order 3 and $\mathbf{A}_{\mathrm{g}}$ is written:

$$
\mathbf{A}_{g}=\frac{\mu}{r^{5}}\left[\begin{array}{ccc}
y^{2}+z^{2} & -x y & -x z \\
-x y & x^{2}+z^{2} & -y z \\
-x z & -y z & x^{2}+y^{2}
\end{array}\right]
$$

with $\mathbf{r}=[x, y, z]^{T}$. Eq. (45) can be rewritten as:

$$
\left\{\begin{array}{l}
\xi_{1}\left(t_{i}\right)=[0,0,0,0,0,0,1]^{T} \\
\dot{\xi}_{1}=\mathbf{A}_{1}(t) \boldsymbol{\xi}_{1}+\mathbf{B}_{1} \mathbf{u}_{l}
\end{array}\right.
$$

with the control vector denoted by $\mathbf{u}_{l}$ and the augmented state $\xi_{1}$ to remove $\mathbf{u}_{0}$ from the equations. The desired optimal control has to minimize the objective function: 


$$
J_{1}\left(\mathbf{u}_{l}\right)=\xi_{1}^{T}\left(t_{f}\right) \mathbf{Q}_{1} \xi_{1}\left(t_{f}\right)+\frac{1}{2} \int_{I}\left\|\mathbf{u}_{l}\right\|^{2} d t
$$

The minimization of (49) provides the feedback control:

$$
\mathbf{u}_{l}=\mathbf{B}_{1}^{T} \mathbf{E} \boldsymbol{\xi}_{1}
$$

where the matrix $\mathbf{E}$ is computed by integrating backwards the Riccati differential equation:

$$
\left\{\begin{array}{l}
\mathbf{E}\left(t_{f}\right)=-\mathbf{Q}_{1} \\
\dot{\mathbf{E}}=-\mathbf{A}_{1}^{T} \mathbf{E}-\mathbf{E} \mathbf{A}_{1}-\mathbf{E} \mathbf{B}_{1} \mathbf{B}_{1}^{T} \mathbf{E}, \forall t \in I_{t}
\end{array}\right.
$$

The first term in the objective function will make $\xi_{l}$ tend towards 0 , which is what is required: the perturbations on the trajectory should not affect the boundaries. The fact that the last component of $\xi_{l}$ is always 1 is not an issue because the choice of $\mathbf{Q}_{1}$ is made such that it does not influence the convergence of the other components of $\boldsymbol{\xi}_{l}$ towards 0. The matrix $\mathbf{Q}_{1}$ is defined as:

$$
\mathbf{Q}_{1}=\left[\begin{array}{ccc}
q_{r} \mathbf{I}_{3} & 0 & 0 \\
0 & q_{v} \mathbf{I}_{3} & 0 \\
0 & 0 & 0
\end{array}\right] \text { for } \quad q_{r}>0, \quad q_{v}>0
$$

where $q_{r}$ is a weight on the final position vector to satisfy the final boundary constraint, and $q_{v}$ has the same role but for the velocity. The values for the two weights were set to 1 in order to satisfy the boundary conditions at arrival up to a relative accuracy of $10^{-6}$. Note that minimizing $J_{1}$ with Eq. (48) is the same as minimizing $J_{l}\left(\mathbf{u}_{l}\right)=\xi^{T}\left(t_{f}\right) \mathbf{Q} \xi\left(t_{f}\right)+\frac{1}{2} \int_{I}\left\|\mathbf{u}_{l}\right\|^{2} d t$ with the condition in Eq. (45). $\mathbf{Q}$ is the matrix composed of the block containing the first six rows and six columns inside $\mathbf{Q}_{1}$.

The optimization requires the integration of a 7 by 7 matrix differential equation backwards in time, followed by the forward integration of the linearized equations of motion using the matrix $\mathbf{E}$. The first integration can be made computationally faster by noting that $\mathbf{E}$ is a symmetric, hence it is sufficient to compute 28 variables instead of 49 . The numerical propagations, in this paper, were performed with the Matlab function ode 45 , that implements a $4^{\text {th }}-5^{\text {th }}$ order Runge-Kutta variable step size integrator, with a relative and absolute tolerance of $10^{-9}$.

Once the optimized linearized trajectory $\mathbf{x}^{*}+\xi$ is computed, the corresponding control law needs to be updated since it verifies the linearized equations of motion and not the real ones. The real control law corresponding to the physical trajectory is calculated from:

$$
\mathbf{u}_{\text {real }}=\ddot{\mathbf{r}}_{\text {real }}+\frac{\mu}{r_{\text {real }}^{3}} \mathbf{r}_{\text {real }}
$$


Note that keeping the linearized control law and calculating the corresponding state vectors by propagation would not only be more computationally intensive but would not guarantee that the trajectory ends at the target state vector. Finally, the total $\Delta v$ can be calculated by an integration of $\mathbf{u}_{\text {real }}$ over $I_{t}$.

\section{B. Estimation of the Error on the Control Profile}

The accuracy of the linearized solution can be assessed by computing the error between $\mathbf{u}_{l}$ and $\mathbf{u}_{\text {real }}$. If $\mathbf{x}_{0}, \mathbf{u}_{0}$ defines the reference trajectory, $\mathbf{x}_{0}+\xi, \mathbf{u}_{l}$ the optimal linearized trajectory and $\mathbf{x}_{0}+\xi, \mathbf{u}_{\text {real }}$ the trajectory obtained after recomputing $\mathbf{u}_{l}$ with the real dynamics, then one has the equations:

$$
\begin{aligned}
& \dot{\mathbf{x}}_{0}=\mathbf{A}\left(\mathbf{x}_{0}\right)+\mathbf{B} \mathbf{u}_{0} \\
& \dot{\boldsymbol{\xi}}=\left.\nabla \mathbf{A}\right|_{\mathbf{x}_{0}} \cdot \boldsymbol{\xi}+\mathbf{B}\left(\mathbf{u}_{l}-\mathbf{u}_{0}\right) \\
& \dot{\mathbf{x}}_{0}+\dot{\boldsymbol{\xi}}=\mathbf{A}\left(\mathbf{x}_{0}+\boldsymbol{\xi}\right)+\mathbf{B} \mathbf{u}_{\text {real }}
\end{aligned}
$$

By subtracting Eq. (54) and (55) from Eq., (56) one gets

$$
\mathbf{B}\left(\mathbf{u}_{l}-\mathbf{u}_{\text {real }}\right)=\mathbf{A}\left(\mathbf{x}_{0}+\boldsymbol{\xi}\right)-\mathbf{A}\left(\mathbf{x}_{0}\right)-\left.\nabla \mathbf{A}\right|_{\mathbf{x}_{0}} \cdot \boldsymbol{\xi}
$$

which can be approximated by:

$$
\mathbf{B}\left(\mathbf{u}_{l}-\mathbf{u}_{\text {real }}\right)=\left.\xi^{T} \mathbf{H}_{\mathbf{A}}\right|_{\mathbf{x}_{0}} \xi+O\left(\|\xi\|^{3}\right)
$$

where $\left.\mathbf{H}_{\mathbf{A}}\right|_{\mathbf{x}_{0}}$ is the Hessian of $\mathbf{A}$ at $\mathbf{x}_{0}$. Because $\mathbf{A}$ depends only on the reference position $\mathbf{r}_{\mathbf{0}},\left.\mathbf{H}_{\mathbf{A}}\right|_{\mathbf{x}_{0}}$ also depends on $\mathbf{r}_{0}$ only. If one defines $\Delta \mathbf{u}=\mathbf{u}_{l}-\mathbf{u}_{\text {real }}=\left[\Delta u_{x}, \Delta u_{y}, \Delta u_{z}\right]^{T}$ and $\xi_{\mathbf{r}}$ as the first three components of $\boldsymbol{\xi}$, i.e., the change of position resulting from the LQ controller, then Eq. (58) can be developed into the system:

$$
\left\{\begin{array}{l}
\Delta u_{x}=\left.\xi_{\mathbf{r}}^{T} \mathbf{H}_{\mathbf{A}}^{1}\right|_{r_{0}} \xi_{\mathbf{r}}+O\left(\left\|\xi_{\mathbf{r}}\right\|^{3}\right) \\
\Delta u_{y}=\left.\xi_{\mathbf{r}}^{T} \mathbf{H}_{\mathbf{A}}^{2}\right|_{r_{0}} \xi_{\mathbf{r}}+O\left(\left\|\xi_{\mathbf{r}}\right\|^{3}\right) \\
\Delta u_{z}=\left.\xi_{\mathbf{r}}^{T} \mathbf{H}_{\mathbf{A}}^{3}\right|_{r_{0}} \xi_{\mathbf{r}}+O\left(\left\|\xi_{\mathbf{r}}\right\|^{3}\right)
\end{array}\right.
$$

In Eqs. (59) one has that all of $\mathbf{H}_{\mathbf{A}}^{i}$ depends only on $\mathbf{r}_{0}$ and that $\left\|\mathbf{H}_{\mathbf{A}}^{i}\right\|=O\left(\left\|\mathbf{r}_{0}\right\|^{-4}\right)$. The interpretation of these equations is that the error on the control law corresponding to the linearized equations of motion depends uniquely on the position of the reference trajectory and the perturbations in position. Moreover, when one assumes that the perturbations in the position are small, then the error behaves as $\left\|\mathbf{r}_{0}\right\|^{-4}$, or as $\left\|\mathbf{r}_{0}\right\|^{-2}$ if one considers the relative perturbations $\xi_{\mathrm{r}} /\left\|\mathbf{r}_{0}\right\|$. Therefore, when the reference trajectory approaches the central body, the control 
corresponding to the real equations of motion diverges from the control computed with the linearized equations of motion.

\section{Optimality of the LQ and shaped solutions}

In this section, it will be proved that: if the shaped solution is locally optimal, then the output from the LQ controller will be equal to the shaped solution. Vice versa, it is demonstrated that when the output of the LQ controller is equal to the shaped solution, the shaped solution is locally optimal.

Let us define the two mathematical problems:

$$
\wp_{l}:=\left\{\begin{array}{c}
\min _{\mathbf{u}_{l}} J_{l}\left(\mathbf{u}_{l}\right) \\
\xi\left(t_{i}\right)=0 \\
\dot{\xi}=f_{l}\left(\xi, \mathbf{u}_{l}\right)=\left.\nabla \mathbf{A}\right|_{\mathbf{x}_{0}(t)} \cdot \boldsymbol{\xi}+\mathbf{B}\left(\mathbf{u}_{l}-\mathbf{u}_{0}\right)
\end{array}\right.
$$

and:

$$
\wp:=\left\{\begin{array}{c}
\min _{\mathbf{u}} J(\mathbf{u}) \\
\mathbf{x}\left(t_{i}\right)=\left[\begin{array}{r}
\mathbf{r}_{0}\left(t_{i}\right), \quad \mathbf{v}_{0}\left(t_{i}\right) \\
{ }^{T}
\end{array}\right. \\
\dot{\mathbf{x}}=f(\mathbf{x}, \mathbf{u})=\mathbf{A}(\mathbf{x})+\mathbf{B u}
\end{array}\right.
$$

where $J$ has the form:

$$
J(\mathbf{u})=\left[\mathbf{x}\left(t_{f}\right)-\mathbf{x}_{0}\left(t_{f}\right)\right]^{T} \mathbf{Q}\left[\mathbf{x}\left(t_{f}\right)-\mathbf{x}_{0}\left(t_{f}\right)\right]+\frac{1}{2} \int_{I}\|\mathbf{u}\|^{2} d t
$$

The Hamiltonians corresponding to $\wp$ and to $\wp_{l}$ are respectively $\quad H=\mathbf{p} f-\|\mathbf{u}\|^{2} / 2$ and $H_{l}=\mathbf{p}_{l} f_{l}-\left\|\mathbf{u}_{l}\right\|^{2} / 2$. If one calls $\mathbf{p}^{*}, \mathbf{u}^{*}, \mathbf{p}_{l}^{*}$ and $\mathbf{u}_{l}^{*}$ the respective optimal adjoint variables and control profiles, then the optimality conditions $\partial H / \partial \mathbf{u}=0$ and $\partial H_{l} / \partial \mathbf{u}_{l}=0$ give the control laws:

$$
\begin{aligned}
& \mathbf{u}^{*}=\mathbf{B}^{T} \mathbf{p}^{* T}=\mathbf{p}_{\mathrm{v}}^{* T} \\
& \mathbf{u}_{l}^{*}=\mathbf{B}^{T} \mathbf{p}_{l}^{* T}=\mathbf{p}_{l v}^{* T}
\end{aligned}
$$

The subscripts $\mathbf{r}$ and $\mathbf{v}$ denote respectively the first three and the last three components of the adjoint vectors. The differential equations governing the optimal adjoint variables $\mathbf{p}^{*}$ and $\mathbf{p}_{l}^{*}$ are:

$$
\begin{aligned}
& \dot{\mathbf{p}}^{*}=-\partial H / \partial \mathbf{x}=-\mathbf{p}^{*} \cdot \partial f / \partial \mathbf{x}=-\left.\mathbf{p}^{*} \cdot \nabla \mathbf{A}\right|_{\mathbf{x}^{*}} \\
& \dot{\mathbf{p}}_{l}^{*}=-\partial H / \partial \xi=-\mathbf{p}_{l}^{*} \cdot \partial f_{l} / \partial \xi=-\left.\mathbf{p}_{l}^{*} \cdot \nabla \mathbf{A}\right|_{\mathbf{x}_{0}(t)}
\end{aligned}
$$

with the transversality conditions: 


$$
\begin{aligned}
& \mathbf{p}^{*}\left(t_{f}\right)=-\left[\mathbf{x}\left(t_{f}\right)-\mathbf{x}_{0}\left(t_{f}\right)\right]^{T} \mathbf{Q}_{0} \\
& \mathbf{p}_{l}^{*}\left(t_{f}\right)=-\boldsymbol{\xi}\left(t_{f}\right)^{T} \mathbf{Q}_{0}
\end{aligned}
$$

From the latter, one gets $\mathbf{p}^{*}\left(t_{f}\right)=\mathbf{p}_{l}^{*}\left(t_{f}\right)$. It is important to note that due to the smoothness of $\mathbf{A}$ and the expression of the differential equations governing $\mathbf{x}^{*}, \mathbf{x}_{l}^{*}, \mathbf{p}^{*}$ and $\mathbf{p}_{l}^{*}$, the last four quantities are $C^{\infty}$ on their interval of definition. Therefore, the control profiles $\mathbf{u}^{*}$ and $\mathbf{u}_{l}^{*}$ are also $C^{\infty}$.

Three lemmas establishing some properties of $\mathbf{A}_{g}$ and three propositions on the nature of the solutions to problems $\wp$ and $\wp_{l}$ are now proved.

Lemma 1: Given the set $U=\mathbb{R}^{3} \backslash\{(0,0,0)\}, \forall \mathbf{x} \in U, \forall \mathbf{q} \in \mathbb{R}^{3}, \mathbf{A}_{g}(\mathbf{x}) \cdot \mathbf{q}=\frac{\mu}{\|\mathbf{x}\|^{5}} \mathbf{x} \times(\mathbf{q} \times \mathbf{x})$.

Proof: Let $\mathbf{x}=[x, y, z]^{-T} \in U$ and $\mathbf{q}=\left[q_{1}, q_{2}, q_{3}\right]^{T} \in \mathbb{R}^{3}$. From the definition of $\mathbf{A}_{g}$, one has:

$$
\mathbf{A}_{g}(\mathbf{x})=\frac{\mu}{\|\mathbf{x}\|^{5}}\left[\begin{array}{ccc}
y^{2}+z^{2} & -x y & -x z \\
-x y & x^{2}+z^{2} & -y z \\
-x z & -y z & x^{2}+y^{2}
\end{array}\right]
$$

then:

$$
\mathbf{A}_{g}(\mathbf{x}) \cdot \mathbf{q}=\frac{\mu}{\|\mathbf{x}\|^{5}}\left(\begin{array}{c}
\left(y^{2}+z^{2}\right) q_{1}-x y q_{2}-x z q_{3} \\
-x y q_{1}+\left(x^{2}+z^{2}\right) q_{2}-y z q_{3} \\
-x z q_{1}-y z q_{2}\left(x^{2}+y^{2}\right) q_{3}
\end{array}\right)
$$

and since

$$
\mathbf{x} \times(\mathbf{q} \times \mathbf{x})=\left(\begin{array}{c}
x \\
y \\
z
\end{array}\right) \times\left(\begin{array}{c}
q_{2} z-q_{3} y \\
q_{3} x-q_{1} z \\
q_{1} y-q_{2} x
\end{array}\right)=\left(\begin{array}{c}
\left(y^{2}+z^{2}\right) q_{1}-x y q_{2}-x z q_{3} \\
-x y q_{1}+\left(x^{2}+z^{2}\right) q_{2}-y z q_{3} \\
-x z q_{1}-y z q_{2}\left(x^{2}+y^{2}\right) q_{3}
\end{array}\right)
$$

then $\mathbf{A}_{g}(\mathbf{x}) \cdot \mathbf{q}=\frac{\mu}{\|\mathbf{x}\|^{5}} \mathbf{x} \times(\mathbf{q} \times \mathbf{x})$.

Lemma 2: $\forall \mathbf{x} \in U$ and $\mathbf{q} \in \mathbb{R}^{3}, \mathbf{A}_{g}(\mathbf{x}) \cdot \mathbf{q}=0 \Leftrightarrow \mathbf{q}$ and $\mathbf{x}$ are collinear.

Proof: From Lemma 1, if one has $\mathbf{A}_{g}(\mathbf{x}) \cdot \mathbf{q}=\mu\|\mathbf{x}\|^{-5} \mathbf{x} \times(\mathbf{q} \times \mathbf{x})=0$, then there exists $\lambda \in \mathbb{R}$ such that $\mathbf{q} \times \mathbf{x}=\lambda \mathbf{x}$. Taking the dot product of both sides with $\mathbf{x}$ in the latter equation and remembering that $\mathbf{x} \neq 0$, one gets $\lambda=0$. Thus $\mathbf{q}$ and $\mathbf{x}$ are collinear. Reciprocally, if $\mathbf{q}$ is collinear with $\mathbf{x}$, then $\mathbf{A}_{g}(\mathbf{x}) \cdot \mathbf{q}=0$. 
Lemma 3: Let $\mathbf{x}, \mathbf{y} \in U$. The matrix $\mathbf{A}_{g}(\mathbf{x})-\mathbf{A}_{g}(\mathbf{y})$ has maximum rank if and only if $\mathbf{x} \neq \pm \mathbf{y}$.

Proof: Let $\mathbf{x}, \mathbf{y} \in U$ such that $\mathbf{x} \neq \pm \mathbf{y}$, and $\mathbf{q} \in \mathbb{R}^{3}$ such that $\mathbf{A}_{g}(\mathbf{x}) \cdot \mathbf{q}=\mathbf{A}_{g}(\mathbf{y}) \cdot \mathbf{q}$, then if $\|\mathbf{x}\|$ and $\|\mathbf{y}\|$ are the Euclidian norms of $\mathbf{x}$ and $\mathbf{y}$ respectively, one has:

$$
\mathbf{x} \times(\mathbf{q} \times \mathbf{x}) /\|\mathbf{x}\|^{5}=\mathbf{y} \times(\mathbf{q} \times \mathbf{y}) /\|\mathbf{y}\|^{5}
$$

Expanding and rearranging this expression to isolate $\mathbf{q}$, one obtains:

$$
\left(\frac{1}{\|\mathbf{x}\|^{3}}-\frac{1}{\|\mathbf{y}\|^{3}}\right) \mathbf{q}=\frac{\mathbf{x} \cdot \mathbf{q}}{\|\mathbf{x}\|^{5}} \mathbf{x}-\frac{\mathbf{y} \cdot \mathbf{q}}{\|\mathbf{y}\|^{5}} \mathbf{y}
$$

If $\|\mathbf{x}\|=\|\mathbf{y}\|$, then Eq.(70) yields $(\mathbf{x} \cdot \mathbf{q}) \mathbf{x}=(\mathbf{y} \cdot \mathbf{q}) \mathbf{y}$ therefore $\mathbf{x}= \pm \mathbf{y}$, which goes against our initial assumption. Therefore $\|\mathbf{x}\| \neq\|\mathbf{y}\|$ and then Eq. (70) results in $\mathbf{q}=\alpha_{\mathbf{q}} \mathbf{x}+\beta_{\mathbf{q}} \mathbf{y}$ with,

$$
\alpha_{\mathbf{q}}=\left(\frac{1}{\|\mathbf{x}\|^{3}}-\frac{1}{\|\mathbf{y}\|^{3}}\right)^{-1} \frac{\mathbf{x} \cdot \mathbf{q}}{\|\mathbf{x}\|^{5}} \quad \beta_{\mathbf{q}}=\left(\frac{1}{\|\mathbf{x}\|^{3}}-\frac{1}{\|\mathbf{y}\|^{3}}\right)^{-1} \frac{\mathbf{y} \cdot \mathbf{q}}{\|\mathbf{y}\|^{5}}
$$

Inserting the expression for $\mathbf{q}$ into Eq. (69) one has, after rearranging the terms:

$$
\left(\frac{\beta_{\mathbf{q}}}{\|\mathbf{x}\|^{5}} \mathbf{x}+\frac{\alpha_{\mathbf{q}}}{\|\mathbf{y}\|^{5}} \mathbf{y}\right) \times(\mathbf{x} \times \mathbf{y})=0
$$

From Eq. (69) it can be obtained that if $\mathbf{x}$ and $\mathbf{y}$ are collinear then $\|\mathbf{x}\|=\|\mathbf{y}\|$. Hence $\mathbf{x}$ and $\mathbf{y}$ are not collinear, i.e., $\mathbf{x} \times \mathbf{y} \neq 0$. The implication is that $\mathbf{q}$ lies in the plane generated by $\mathbf{x}$ and $\mathbf{y}$. Eq.(72) results in $\beta_{\mathbf{q}}\|\mathbf{x}\|^{-5} \mathbf{x}+\alpha_{\mathbf{q}}\|\mathbf{y}\|^{-5} \mathbf{y}=0$, thus $\quad \alpha_{\mathbf{q}}=\beta_{\mathbf{q}}=0 \quad$ and $\quad \mathbf{x} \cdot \mathbf{q}=\mathbf{y} \cdot \mathbf{q}=0$ which implies $\mathbf{q}=0$. Therefore the endomorphism $\mathbf{A}_{g}(\mathbf{x})-\mathbf{A}_{g}(\mathbf{y})$ is injective and hence invertible, and its rank is 3 .

Conversely, it can be checked that if $\mathbf{x}= \pm \mathbf{y}$, then $\mathbf{A}_{g}(\mathbf{x})=\mathbf{A}_{g}(\mathbf{y})$ and $\mathbf{A}_{g}(\mathbf{x})-\mathbf{A}_{g}(\mathbf{y})$ does not have maximum rank.

Proposition 1: Let $\mathbf{u}^{*}$ (respectively $\mathbf{u}_{l}^{*}$ ) be a solution of the optimization problem $\wp \quad\left(\right.$ resp. $\left.\wp_{l}\right)$. Then $\mathbf{p}^{*_{T}}=\left[\begin{array}{lll}-\dot{\mathbf{u}}^{*_{T}} & \mathbf{u}^{*_{T}} & \kappa\end{array}\right]^{T}$ (resp. $\mathbf{p}_{l}^{*_{T}}=\left[\begin{array}{lll}-\dot{\mathbf{u}}_{l}^{*_{T}} & \mathbf{u}_{l}^{{ }^{*} T} & \kappa_{l}\end{array}\right]^{T}$ ), where $\kappa$ (resp. $\left.\kappa_{l}\right)$ is a scalar function, and $\mathbf{u}^{*}$ (resp. $\mathbf{u}_{l}^{*}$ ) satisfies the differential equations: 


$$
\begin{aligned}
& \ddot{\mathbf{u}}^{*}+\mathbf{A}_{g}\left(\left(\mathbf{x}_{0}+\xi\right)(t)\right) \mathbf{u}^{*}=0 \\
& \ddot{\mathbf{u}}_{l}^{*}+\mathbf{A}_{g}\left(\mathbf{x}_{0}(t)\right) \mathbf{u}_{l}^{*}=0
\end{aligned}
$$

Proof: From the optimality conditions, one has the expressions $\mathbf{u}^{*}=\mathbf{B}_{1}^{T} \mathbf{p}^{{ }^{*} T}$ and $\mathbf{u}_{l}^{*}=\mathbf{B}_{1}^{T} \mathbf{p}_{l}^{* T}$. Developing these expressions, the control vectors correspond to the fourth to sixth components of the adjoint variables. Moreover, since $\dot{\mathbf{p}}^{* T}=-\mathbf{A}_{1}^{T}\left(\left(\mathbf{x}_{0}+\boldsymbol{\xi}\right)(t)\right) \mathbf{p}^{* T}$ and $\dot{\mathbf{p}}_{l}^{* T}=-\mathbf{A}_{1}^{T}\left(\mathbf{x}_{0}(t)\right) \mathbf{p}_{l}^{* T}$, one gets $\dot{\mathbf{u}}^{*}=\mathbf{B}_{1}^{T} \dot{\mathbf{p}}^{* T}=-\mathbf{B}_{1}^{T} \mathbf{A}_{1}^{T}\left(\left(\mathbf{x}_{0}+\boldsymbol{\xi}\right)(t)\right) \mathbf{p}^{* T}$ and $\dot{\mathbf{u}}_{l}^{*}=\mathbf{B}_{1}^{T} \dot{\mathbf{p}}_{l}^{* T}=-\mathbf{B}_{1}^{T} \mathbf{A}_{1}^{T}\left(\mathbf{x}_{0}(t)\right) \mathbf{p}_{l}^{* T}$. By developing the expressions of $\mathbf{A}_{1}^{T}$ and $\mathbf{B}_{1}^{T}$, one finds that the first three components of the adjoint variables correspond to the opposite of the derivative of the controls. So one can write $\mathbf{p}^{{ }^{*} T}=\left[\begin{array}{lll}-\dot{\mathbf{u}}^{{ }^{*} T} & \mathbf{u}^{{ }^{*} T} & \kappa\end{array}\right]^{T}$ and $\mathbf{p}_{l}^{{ }^{*} T}=\left[\begin{array}{lll}-\dot{\mathbf{u}}_{l}{ }^{*} & \mathbf{u}_{l}^{{ }^{*} T} & \kappa_{l}\end{array}\right]^{T}$. From the expressions of the derivatives of the adjoint variables, one obtains:

$$
\begin{gathered}
\dot{\mathbf{p}}^{* T}=\left(\begin{array}{c}
-\ddot{\mathbf{u}}^{*} \\
\dot{\mathbf{u}}^{*} \\
\dot{\kappa}
\end{array}\right)=-\mathbf{A}_{1}^{T}\left(\left(\mathbf{x}_{0}+\boldsymbol{\xi}\right)(t)\right)\left(\begin{array}{c}
-\dot{\mathbf{u}}^{*} \\
\mathbf{u}^{*} \\
\kappa
\end{array}\right)=\left(\begin{array}{c}
-\mathbf{A}_{g}\left(\left(\mathbf{x}_{0}+\boldsymbol{\xi}\right)(t)\right) \mathbf{u}^{*} \\
\dot{\mathbf{u}}^{*} \\
\mathbf{u}_{0}^{T} \mathbf{u}^{*}
\end{array}\right) \\
\dot{\mathbf{p}}_{l}^{* T}=\left(\begin{array}{c}
-\ddot{\mathbf{u}}_{l}^{*} \\
\dot{\mathbf{u}}_{l}^{*} \\
\dot{\kappa}_{l}^{*}
\end{array}\right)=-\mathbf{A}_{1}^{T}\left(\mathbf{x}_{0}(t)\right)\left(\begin{array}{c}
-\dot{\mathbf{u}}_{l}^{*} \\
\mathbf{u}_{l}^{*} \\
\boldsymbol{\kappa}_{l}
\end{array}\right)=\left(\begin{array}{c}
-\mathbf{A}_{g}\left(\mathbf{x}_{0}(t)\right) \mathbf{u}_{l}^{*} \\
\dot{\mathbf{u}}_{l}^{*} \\
\mathbf{u}_{0}^{T} \mathbf{u}_{l}^{*}
\end{array}\right)
\end{gathered}
$$

The differential equations satisfied by the components of $\mathbf{u}^{*}$ and $\mathbf{u}_{l}^{*}$ are the first three components in Eqs. (74) and (75).

Proposition 2: Let $\mathbf{u}^{*}$ (respectively $\mathbf{u}_{l}^{*}$ ) be a solution of the optimization problem $\wp \quad\left(\right.$ resp. $\left.\wp_{l}\right)$ ). Let $I_{t}=\left(\begin{array}{ll}t_{1} & t_{2}\end{array}\right) \subset \mathbb{R}$ be an open interval of time. Let us assume that the angular momentum along the trajectory corresponding to $\mathbf{u}^{*}\left(\right.$ resp. $\left.\mathbf{u}_{0}\right)$ is never zero. If $\mathbf{p}_{\mathbf{r}}^{*}=0\left(\right.$ resp. $\left.\mathbf{p}_{l \mathbf{r}}^{*}=0\right)$ on $I_{t}$, then $\mathbf{u}^{*}=0\left(\right.$ resp. $\left.\mathbf{u}_{l}^{*}=0\right)$ on $I_{t}$.

Proof: From proposition 1, $\mathbf{p}_{\mathbf{r}}^{*}=0\left(\right.$ resp. $\left.\mathbf{p}_{l \mathrm{r}}^{*}=0\right)$ on $I_{t}$ implies that $\dot{\mathbf{u}}^{*}=0\left(\right.$ resp. $\left.\dot{\mathbf{u}}_{l}^{*}=0\right)$ on $I_{t}$, and thus $\ddot{\mathbf{u}}^{*}=0$ (resp. $\left.\ddot{\mathbf{u}}_{l}^{*}=0\right)$ on $I_{t}$. From the differential equations provided by Proposition 1, one obtains $\mathbf{A}_{g}\left(\left(\mathbf{x}_{0}+\boldsymbol{\xi}\right)(t)\right) \mathbf{u}^{*}=0$ (resp. $\left.\mathbf{A}_{g}\left(\mathbf{x}_{0}(t)\right) \mathbf{u}_{l}^{*}=0\right)$. Thus, according to Lemma 2, there exists a scalar function $\lambda$ on $I_{t}$, such that $\mathbf{u}^{*}=\lambda\left(\mathbf{x}_{\mathrm{r} 0}+\boldsymbol{\xi}_{\mathrm{r}}\right)\left(\right.$ resp. $\left.\mathbf{u}_{l}^{*}=\lambda \mathbf{x}_{\mathrm{r} 0}\right) . \lambda$ is continuously differentiable because $\mathbf{x}_{\mathrm{r} 0}+\xi_{\mathrm{r}}$ (resp. $\left.\mathbf{x}_{\mathrm{r} 0}\right)$ is continuously differentiable. One obtains then the differential equation $\dot{\lambda}\left(\mathbf{x}_{\mathrm{r} 0}+\boldsymbol{\xi}_{\mathrm{r}}\right)+\lambda\left(\dot{\mathbf{x}}_{\mathrm{r} 0}+\dot{\boldsymbol{\xi}}_{\mathrm{r}}\right)=0 \quad\left(\right.$ resp. $\left.\quad \dot{\lambda}_{\mathbf{r} 0}+\lambda \dot{\mathbf{x}}_{\mathrm{r} 0}=0\right)$. Because the trajectories are always assumed to have angular momentum bounded away from zero, one gets $\lambda=0$ and $\dot{\lambda}=0$ and thus $\mathbf{u}^{*}=0$ (resp. $\mathbf{u}_{l}^{*}=0$ ) on $I_{t}$. Due to the continuous nature of the optimal thrust profiles, the latter result is valid on $\bar{I}_{t}=\left[\begin{array}{ll}t_{1} & t_{2}\end{array}\right]$. 
Proposition 3: Let $\mathbf{u}^{*}$ be a solution of $\wp$ and $\mathbf{u}_{l}^{*}$ a solution of $\wp_{l}$. Let us assume that the angular momentum of the initial trajectory $\mathbf{x}_{0}$ never cancels. If $\mathbf{u}^{*}=\mathbf{u}_{l}^{*}$ then there are three regimes in which the trajectory can evolve:
1) $\mathbf{x}^{*}=\mathbf{x}_{0}$
2) $\mathbf{x}^{*}=-\mathbf{x}_{0}$
3) $\mathbf{u}^{*}=\mathbf{u}_{l}^{*}=0$, i.e., the optimal trajectories are coast arcs.

Moreover, the optimal trajectories cannot switch between regimes 1 and 2 without passing through regime 3 on an open interval of time $\left(\begin{array}{ll}t_{1} & t_{2}\end{array}\right)$, and switching between regime 1 and regime 3 can only happen if $\mathbf{u}_{0}=0$ at the boundary.

Proof: From Eqs. (63), if $\mathbf{u}^{*}=\mathbf{u}_{l}^{*}$ then $\mathbf{p}_{\mathrm{v}}^{*}=\mathbf{p}_{l v}^{*}$ and $\dot{\mathbf{p}}_{\mathrm{v}}^{*}=\dot{\mathbf{p}}_{l v}^{*}$. Furthermore, $\dot{\mathbf{p}}_{\mathrm{v}}^{*}=-\mathbf{p}_{\mathrm{r}}^{*}$ and $\dot{\mathbf{p}}_{l v}^{*}=-\mathbf{p}_{l \mathrm{r}}^{*}$ implies $\mathbf{p}_{\mathbf{r}}^{*}=\mathbf{p}_{l \mathbf{r}}^{*}$, thus $\mathbf{p}^{*}=\mathbf{p}_{l}^{*}$. From Eqs.(64) one gets $\mathbf{p}_{l}^{*} \cdot\left(\left.\nabla \mathbf{A}\right|_{\mathbf{x}^{*}}-\left.\nabla \mathbf{A}\right|_{\mathbf{x}_{0}}\right)=0$ therefore $\mathbf{p}_{l \mathbf{r}}^{*} \cdot\left[\mathbf{A}_{g}\left(\mathbf{x}^{*}\right)-\mathbf{A}_{g}\left(\mathbf{x}_{0}\right)\right]=0$. According to Lemma $3, \mathbf{A}_{g}\left(\mathbf{x}^{*}\right)-\mathbf{A}_{g}\left(\mathbf{x}_{0}\right)$ has full rank as long as $\mathbf{x}^{*} \neq \pm \mathbf{x}_{0}$, therefore, there exists three regimes in which the trajectory can evolve: $\mathbf{x}^{*}=\mathbf{x}_{0}, \mathbf{x}^{*}=-\mathbf{x}_{0}$ or $\mathbf{p}_{l \mathbf{r}}^{*}=0$. Proposition 2 can be applied for regime 3 because the angular momentum of the initial trajectory $\mathbf{x}_{0}$ is assumed to never cancel. If $\mathbf{p}_{l \mathbf{r}}^{*}=0$ on an interval of time $\left(\begin{array}{ll}t_{1} & t_{2}\end{array}\right)$, then $\mathbf{u}^{*}=\mathbf{u}_{l}^{*}=0$ on $\left(\begin{array}{ll}t_{1} & t_{2}\end{array}\right)$, i.e. the optimal trajectories are coast arcs.

The trajectory cannot switch between regime 1 and regime 2 directly because the trajectory is continuous. Therefore, regimes 1 and 2 can switch only if regime 3 takes place between the two. However, in that case, the system has to be in regime 3 on an interval of time $\left(\begin{array}{ll}t_{1} & t_{2}\end{array}\right)$ and not for an isolated instant of time $t_{0}$, because otherwise, due to the continuity of the trajectory, the system would bounce back to the regime leading up to regime 3 . There is a condition when the system can swap between regime 1 and regime 3 on an interval of time $\left(\begin{array}{ll}t_{1} & t_{2}\end{array}\right)$ since at the boundary between regime 1 and regime $3 \xi=0$ and $\dot{\xi}=0$, from Eq. (45) one obtains that $\mathbf{u}_{l}^{*}=\mathbf{u}_{0}$ at the switching point, otherwise the velocity profile would not be continuous. Finally, because when the system is in regime 3 on $I_{t}=\left(\begin{array}{ll}t_{1} & t_{2}\end{array}\right), \mathbf{u}^{*}=\mathbf{u}_{l}^{*}=0$ on $I$, by continuity on the controls are zero on the closure of $I$, i.e.,on $\bar{I}_{t}=\left[\begin{array}{ll}t_{1} & t_{2}\end{array}\right]$, and finally one obtains that at the boundary between regimes 1 and $3, \mathbf{u}_{0}=0$.

Theorem: Let $\mathbf{u}^{*}$ be a solution of $\wp$ and $\mathbf{u}_{l}^{*}$ a solution of $\wp_{l}$, then $\mathbf{u}^{*}=\mathbf{u}_{0} \Rightarrow \mathbf{u}^{*}=\mathbf{u}_{l}^{*}$. Furthermore, if $\mathbf{u}_{0} \neq 0$ along the whole trajectory and the angular momentum of the reference trajectory $\mathbf{x}_{0}$ is never zero, then $\mathbf{u}^{*}=\mathbf{u}_{l}^{*} \Rightarrow \mathbf{u}^{*}=\mathbf{u}_{0}$. 
Proof: The first inference is proven first. If $\mathbf{u}^{*}=\mathbf{u}_{0}$, then $\mathbf{x}^{*}=\mathbf{x}_{0}$ and the equations governing $\mathbf{p}^{*}$ and $\mathbf{p}_{l}^{*}$ are identical, thus $\mathbf{p}^{*}=\mathbf{p}_{l}^{*}$ and $\mathbf{u}^{*}=\mathbf{u}_{l}^{*}$. Note that to establish this inference, no particular property of $\nabla \mathbf{A}$ is required.

The proof of the reciprocal inference requires special properties of the dynamical systems, and therefore of the gravity field. This inference is a corollary of Proposition 3. Because the starting points of the trajectories are fixed at $\mathbf{x}^{*}\left(t_{i}\right)=\mathbf{x}_{l}^{*}\left(t_{i}\right)=\mathbf{x}_{0}\left(t_{i}\right)$, the optimized trajectories start in regime 1 and since $\mathbf{u}_{0} \neq 0$ along the whole trajectory, the system remains in the same regime all the time. Therefore, $\mathbf{x}^{*}=\mathbf{x}_{0}$ and $\mathbf{u}^{*}=\mathbf{u}_{0}$ along the whole trajectory.

From this result one could argue that if the LQ controller does not modify the reference trajectory, then the reference trajectory is locally optimal, on the other hand, little can be said if the control $\mathbf{u}_{l}^{*}$ is worse than $\mathbf{u}_{0}$.

\section{Application of the LQ Controller}

The LQ controller is applied to the improvement of the solutions to the test cases presented in Section II.E. Only those transfers that do not pass inside Venus's orbit were retained in order to keep the error due to linearization small. Fig. 8 to Fig. 11 show the improvement of the $L_{2}$ norm of the control profiles $\frac{1}{2} \int_{I}\|\mathbf{u}\|^{2} d t$ between the shaped trajectories and the corresponding LQ controlled trajectories.
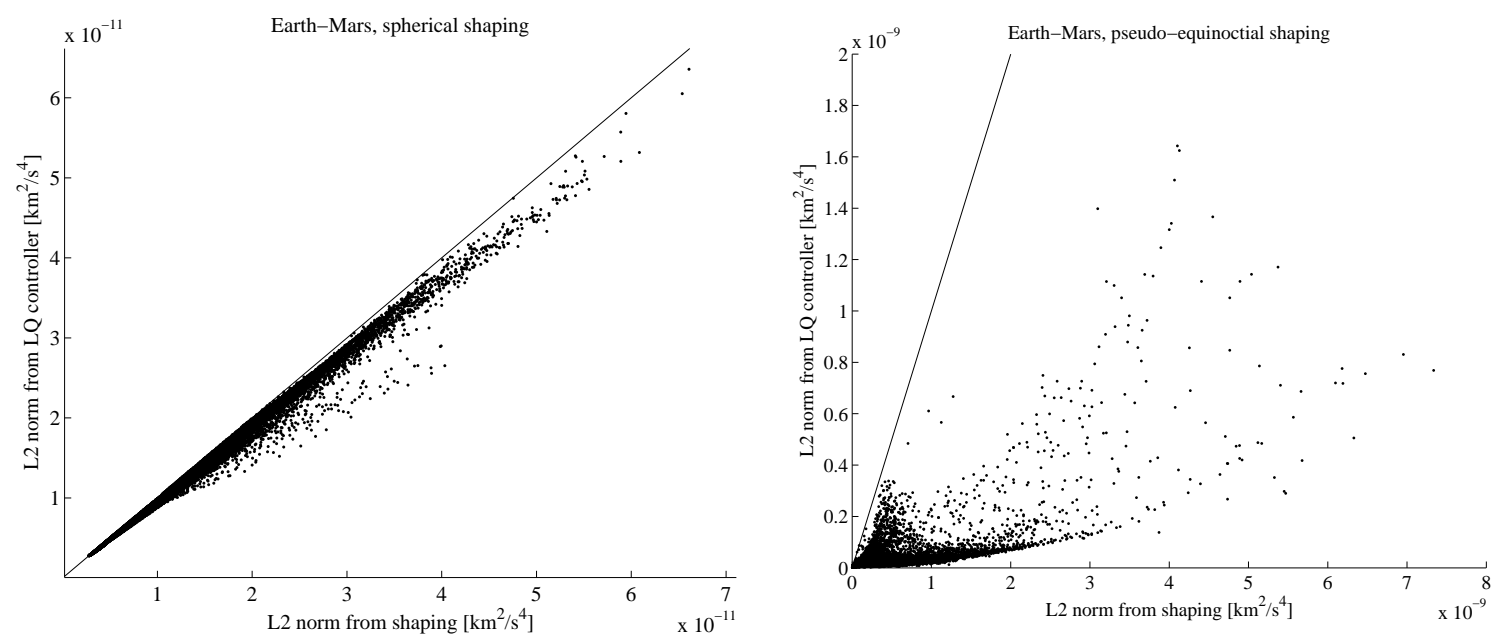

Fig. 8: Comparison between the $L_{2}$ norms of the controls of the spherical and pseudo-equinoctial shaped transfers and the corresponding LQ-controlled improvement for the Mars rendezvous mission. 

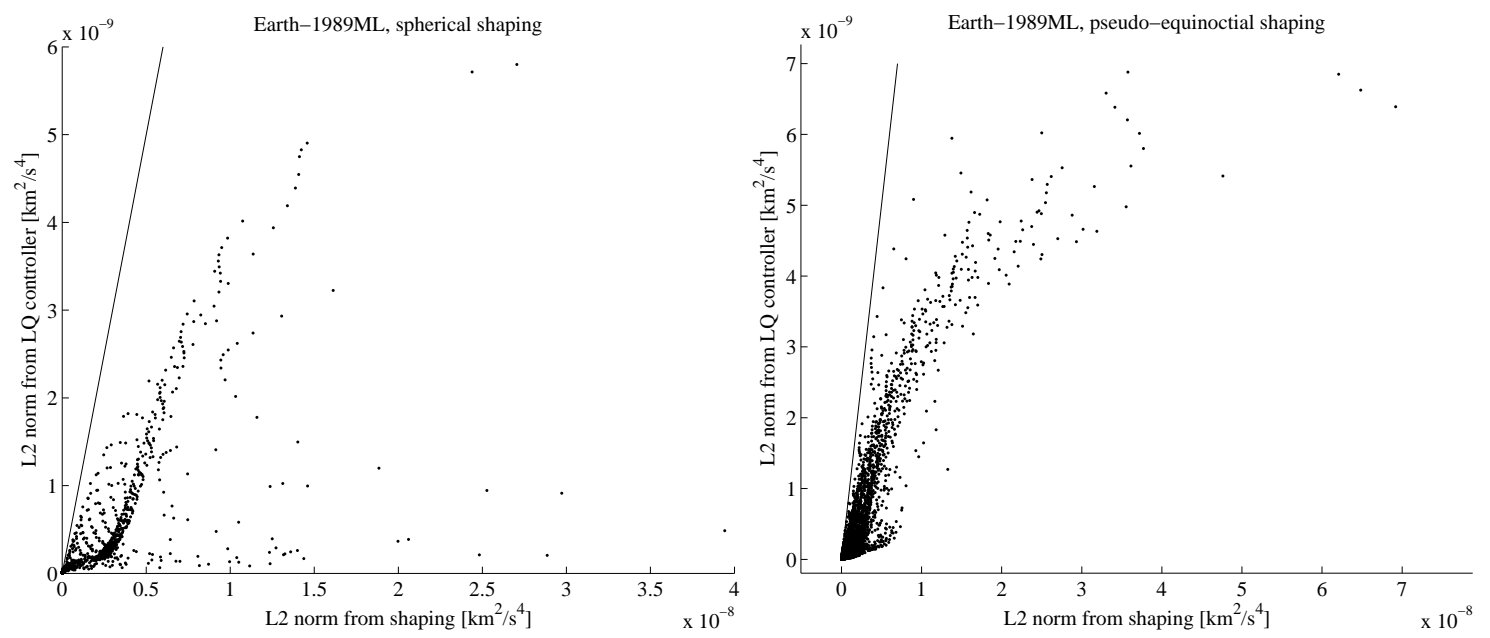

Fig. 9: Comparison between the $L_{2}$ norms of the controls of the spherical and pseudo-equinoctial shaped transfers and the corresponding LQ-controlled improvmement for the 1989ML rendezvous mission.
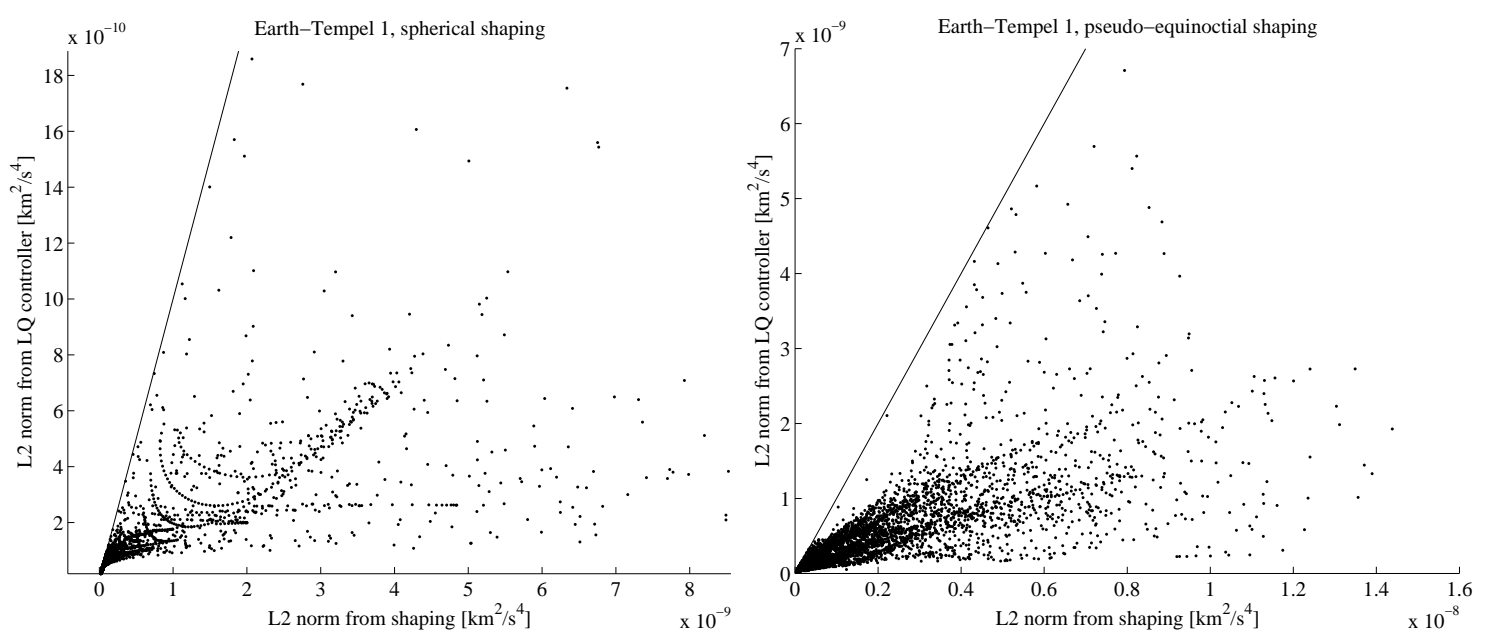

Fig. 10: Comparison between the $L_{2}$ norms of the controls of the spherical and pseudo-equinoctial shaped transfers and the corresponding LQ-controlled improvement for the Tempel 1 rendezvous mission. 

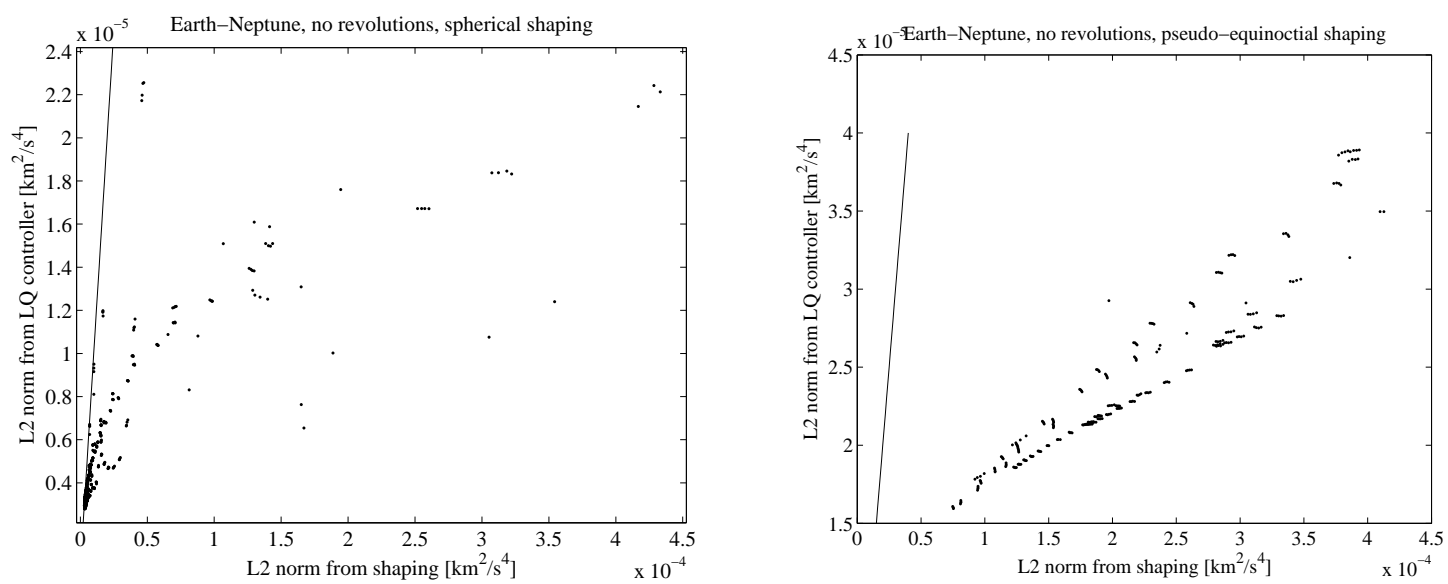

Fig. 11: Comparison between the $L_{2}$ norms of the controls of the spherical and pseudo-equinoctial shaped transfers and the corresponding LQ-controlled improvement for the Neptune rendezvous mission, without revolutions.

The typical way for a mission analyst to proceed would be to perform a search on a wide search search space using the combination of a shaping approach and the LQ controller. Promising individual trajectories can then be further optimized with a local optimizer like DITAN, which is fed with the control profile of the initial guess.

Fig. 12 to Fig. 17 are examples of thrust profiles corresponding to the shaped trajectories, the LQ controlled trajectories and the DITAN re-optimized trajectories, for all rendezvous missions. In this case, DITAN was minimizing the $L_{2}$ norm of the control in order to assess the optimality of the control profile provided by the LQ controller. No upper limit was set on the control magnitude. The figures illustrate well how the control profiles improve at each step.

It can be seen that the peak thrusts are reduced in all cases, except in the case of the transfer to 1989ML with the pseudo-equinoctial shaping used as trajectory generator. Fig. 13, Fig. 16 and Fig. 17 show particularly large reductions in the peak thrust by the LQ controller. The general features of the thrust profile resulting from the LQ controller are always closer to the optimal one from DITAN. The LQ controller adjusts the maneuvers such that they tend to take place at places where they are more efficient. The example of the Mars rendezvous in Fig. 12 shows thrust profiles from the LQ controller and DITAN that are very similar. The reason is that the initial shaped trajectory is itself not far from the optimal one. 

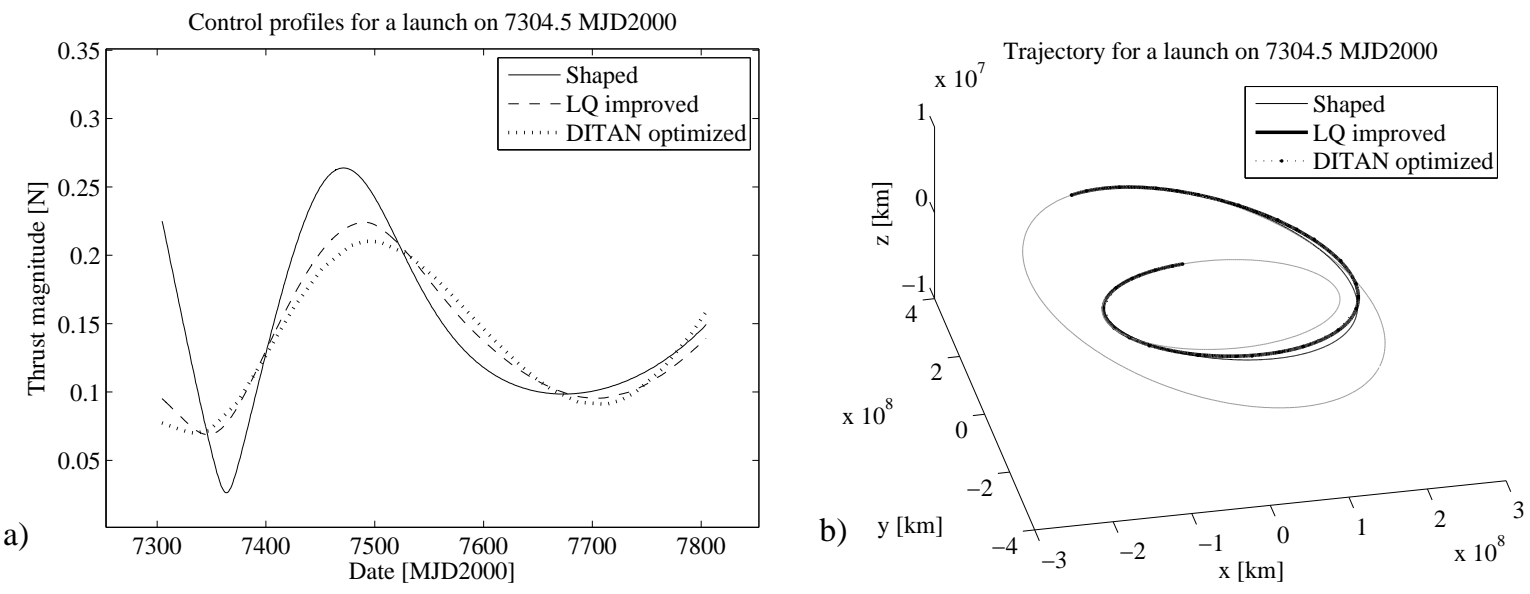

Fig. 12: Rendezvous mission to Mars. Comparison between spherical shaped solution, LQ optimized solution and DITAN optimized solution: a) control profile, b) trajectory.
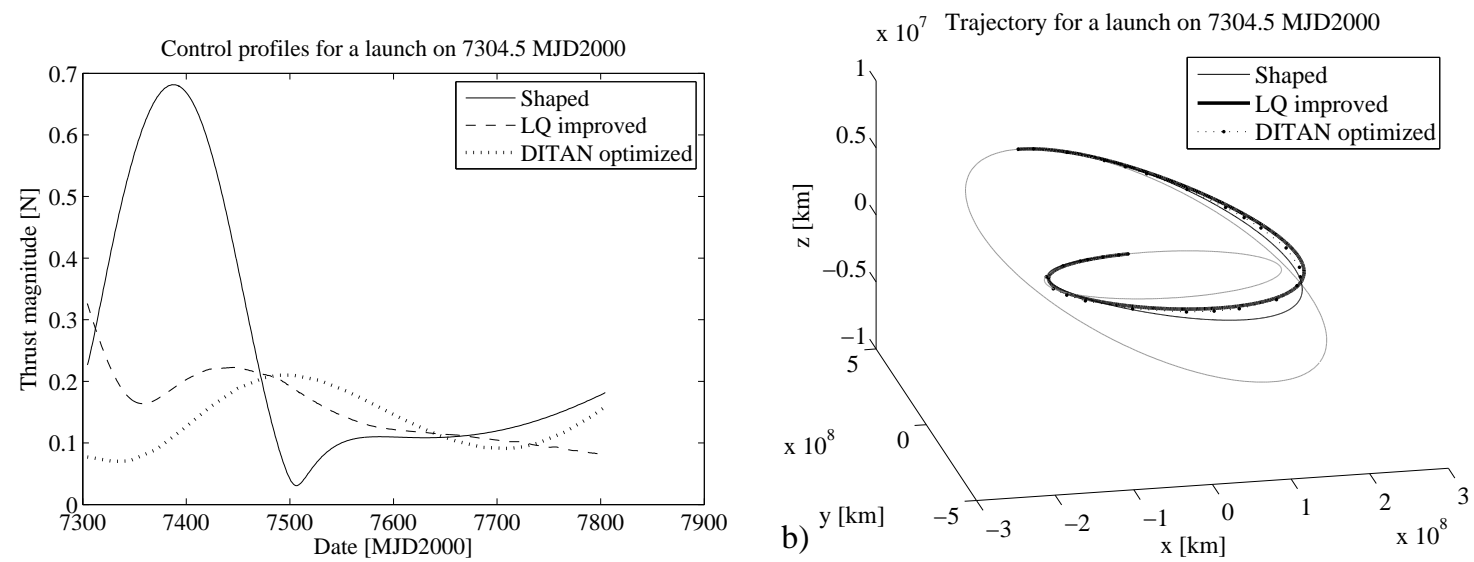

Fig. 13: Rendezvous mission to Mars. Comparison between pseudo-equinoctial shaped solution, LQ optimized solution and DITAN optimized solution: a) control profile, b) trajectory.

a)

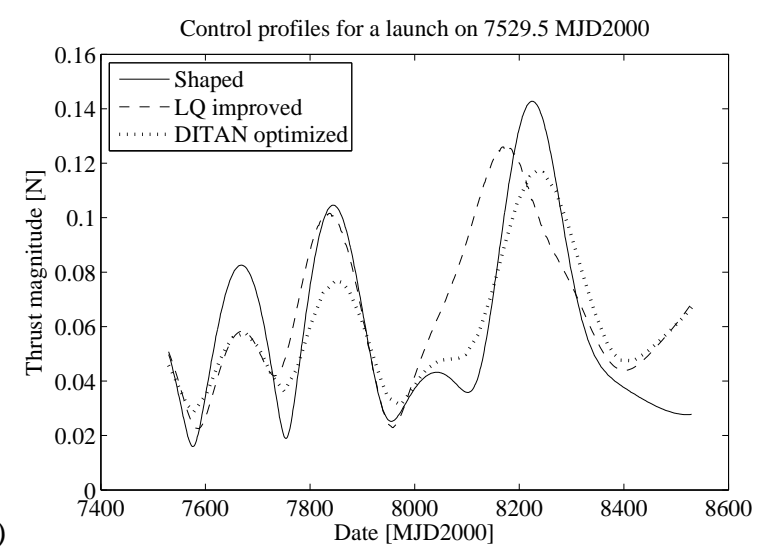

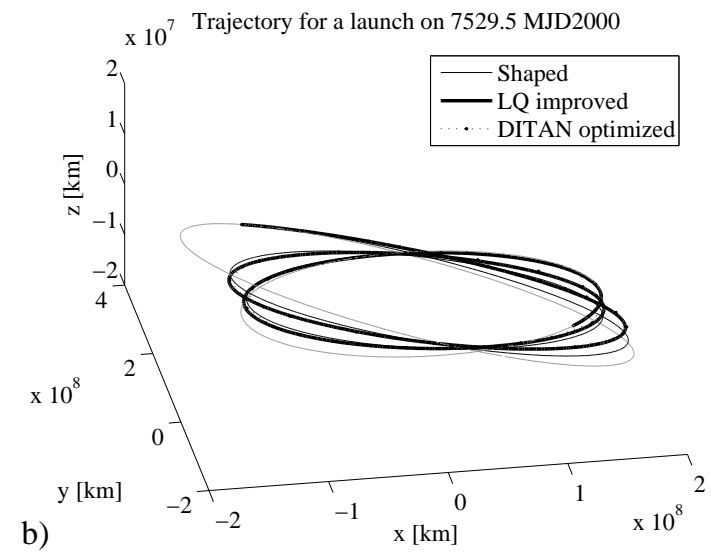

b)

Fig. 14: Rendezvous mission to asteroid 1989ML. Comparison between spherical shaped solution, LQ optimized solution and DITAN optimized solution: a) control profile, b) trajectory. 
a)
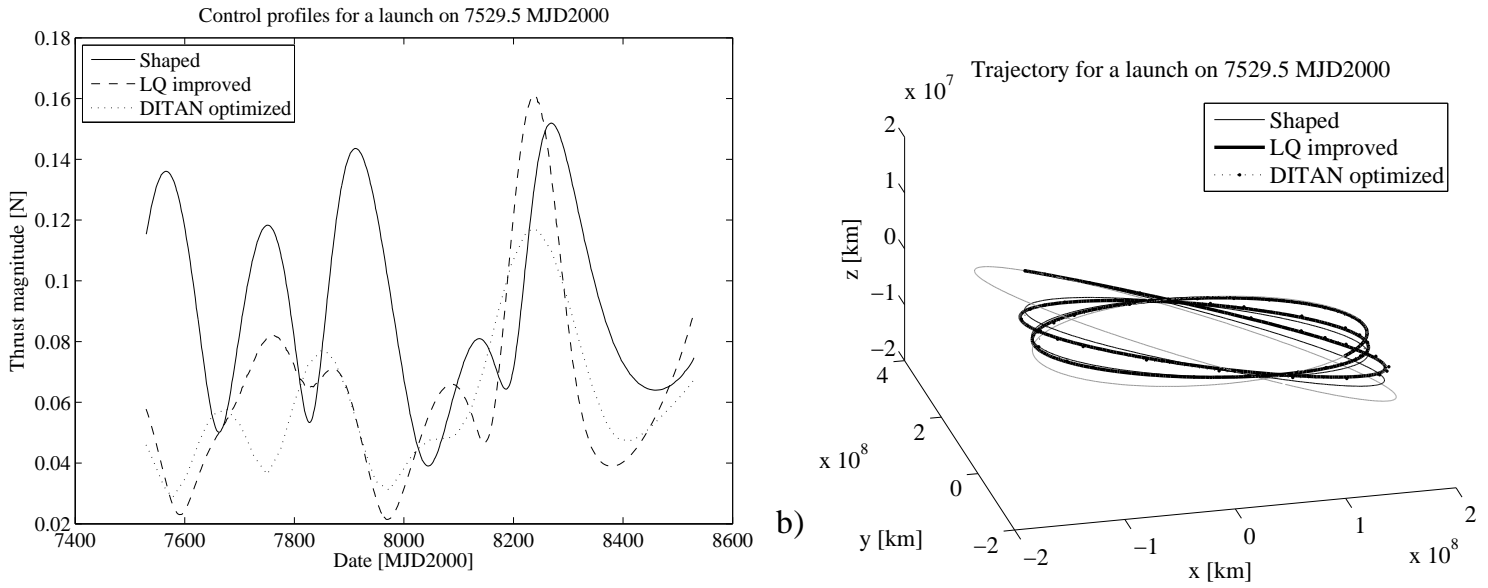

Fig. 15: Rendezvous mission to asteroid 1989ML. Comparison between pseudo-equinoctial shaped solution, LQ optimized solution and DITAN optimized solution: a) control profile, b) trajectory.

a)
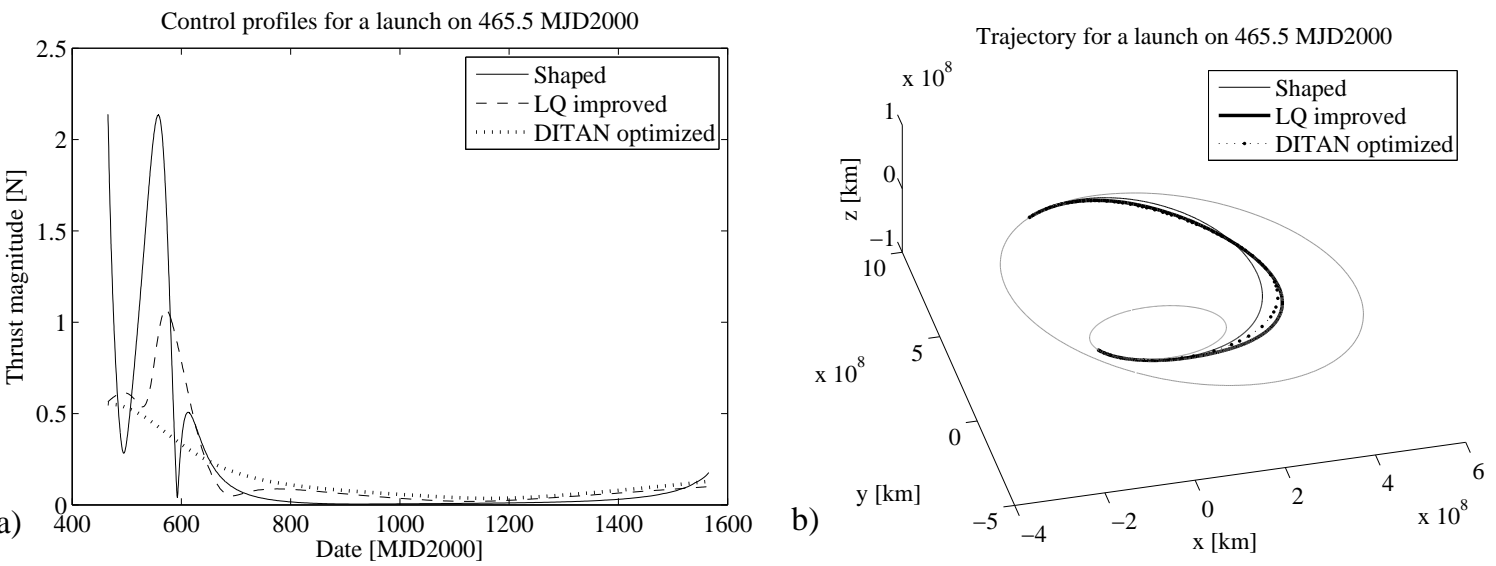

Fig. 16: Rendezvous mission to Tempel 1. Comparison between spherical shaped solution, LQ optimized solution and DITAN optimized solution: a) control profile, b) trajectory.
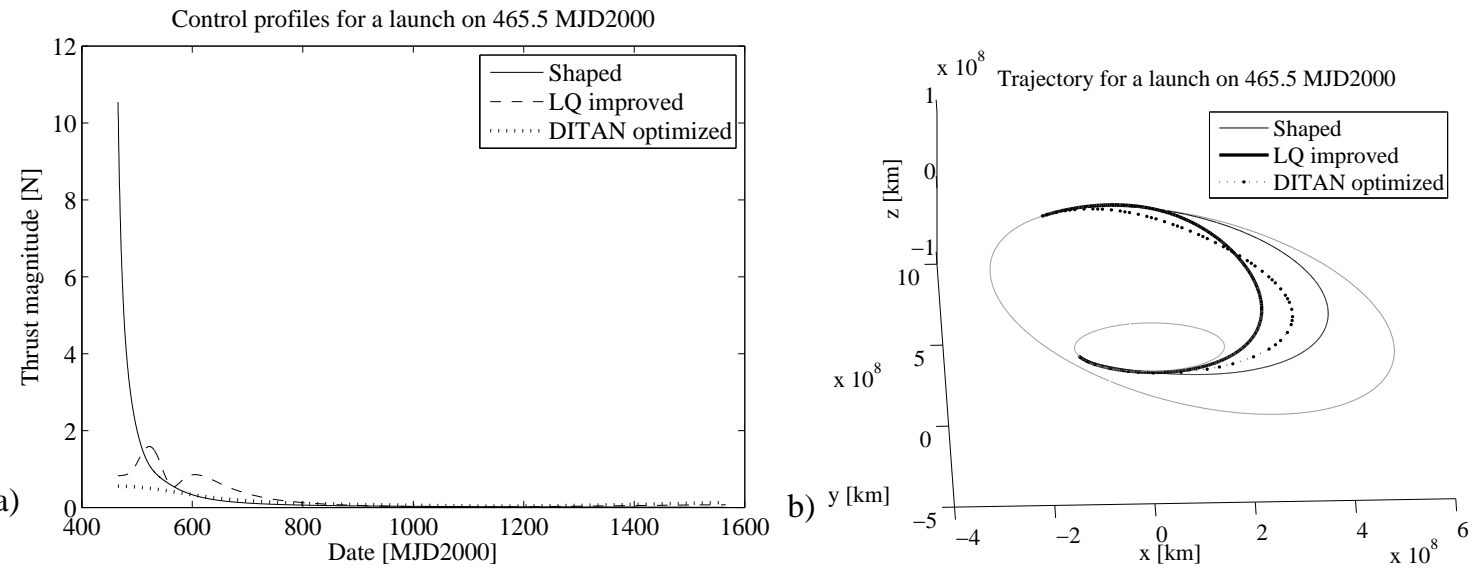
Fig. 17: Rendezvous mission to Tempel 1. Comparison between pseudo-equinoctial shaped solution, LQ optimized solution and DITAN optimized solution: a) control profile, b) trajectory.

Fig. 18 illustrates the thrust profiles and the trajectories corresponding to a rendezvous transfer to Neptune. The plots include the results from the spherical shaping, the LQ controller and DITAN for minimal $L_{2}$ norm of the thrust and minimal propellant mass. The figures show that the result from the shaping is closer to the optimal propellant mass solution than to the $L_{2}$-norm one. The thrust profile of the shaped trajectory is close to being bang-off-bang and the optimal propellant mass trajectory is very similar. Unlike the shaped solution, the optimal one presents a small maneuver towards the end of the transfer for a plane change. In fact, a plane change maneuver is more efficient when performed far from the Sun. Note that the switching structure from DITAN would be better defined with a finer grid.

a)

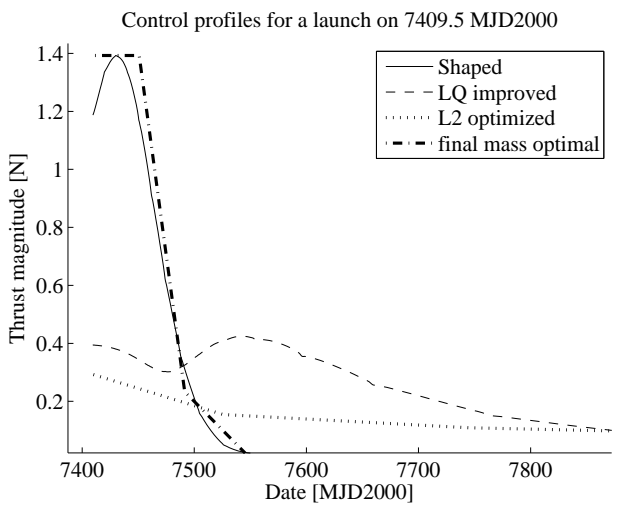

Control profiles for a launch on 7409.5 MJD2000

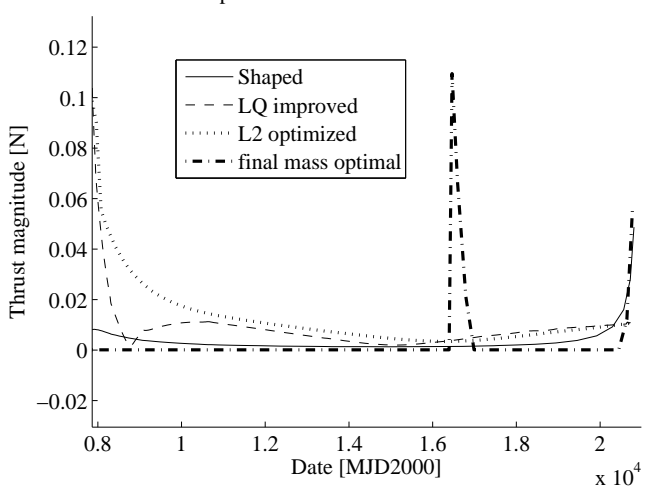

c)

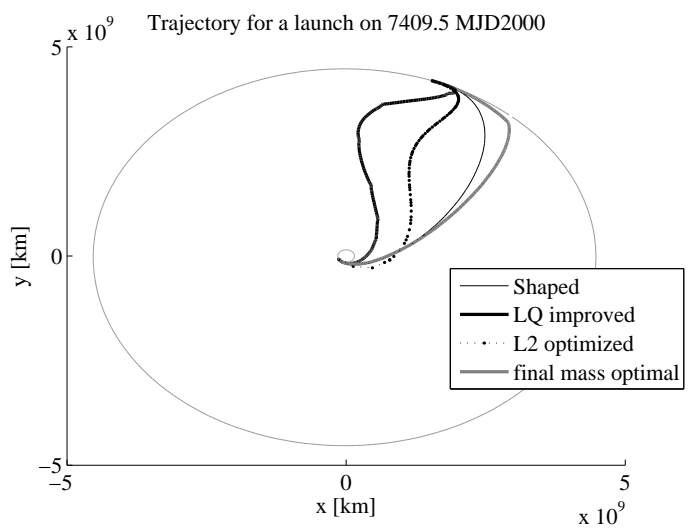

Fig. 18: Rendezvous mission to Neptune, with an initial tangential relative velocity of $3 \mathrm{~km} / \mathrm{s}$. Comparison between spherical shaped solution, LQ optimized solution and DITAN optimized solution: a) first part of control profile, b) second part of control profile, c) trajectory. 
In some cases, there is no improvement of the $L_{2}$ norm of the control. This happens when the trajectory is too close to the central body, in which case the control corresponding to the real equations of motion diverges from the optimal control of the linearized equations of motion, as described in technical terms in Section II.B. Otherwise, the LQ controller has the tendency to reduce the control magnitude because a better $L_{2}$ norm of the control translates, in general, to lower peak controls. Applying the LQ controller to shaped trajectories can therefore reduce the risk of discarding some mission scenarios due to the high magnitude of the peak thrust.

\section{E. Computational times}

Table 6 summarizes the computational times required by each tool used in the present study. The shaping methods require a fraction of a second, depending on the number of times the trajectory is recomputed within the Newton loop in order to satisfy the time of flight constraints. The computational effort required by the LQ controller is generally one order of magnitude higher, i.e. seconds, than the trajectories generated by shaping. The calculation time depends on the length of the trajectory, and the tolerances used to integrate the Riccati differential equation and the equations of motion. This time also depends on how many points are used to define the reference; the more points provided along the trajectory, the more time the interpolations require for the integrators to calculate each step. The speed of convergence of the low thrust optimizer DITAN generally depends on the initial thrust profile, and varies between 20 and 100 seconds. A better initial guess will generally reduce the computational time.

Table 6: Computational effort required by the different trajectory generating and improving tools used in this study.

\begin{tabular}{cc}
\hline \hline & Computational time [s] \\
\hline Shaping methods & $\sim 0.1-0.3$ \\
LQ controller & $\sim 2-6$ \\
DITAN optimizer & $\sim 20-100$ \\
\hline \hline
\end{tabular}

\section{Conclusion}

This paper proposed a method to quickly generate approximated low-thrust solutions by combining a novel shaping method with an LQ controller. The proposed approach provides transfers satisfying the boundary conditions with practically acceptable control magnitudes at a low computational cost. The novel shaping method was derived from a mathematical framework that generalizes the existing results in the literature. The pseudo-equinoctial shaping was revisited within the unifying framework, providing conditions to derive osculating shaped parameters describing low-thrust trajectories. The case studies presented in this paper prove that the novel shaping in spherical coordinates provides, on average, better solutions than the pseudo-equinoctial shaping. For a number of transfers, the shaped solution is very close to the fully optimized one. When combined with the LQ controller, this shaping 
method can offer a clear decision criterion on the feasibility of a large number of transfer solutions for any two given orbits. Although the applicability of the LQ controller is limited by the distance from the center of gravity, initial experiments demonstrate that this limitation can be removed by iterating the LQ controller for a limited number of times with little overhead.

\section{Appendix A}

This Appendix presents the derivations for the expressions of $\tilde{\mathbf{v}}$ and $\tilde{\mathbf{a}}$ in different reference frames. The resulting expressions are used in particular in the computation of $D$ in (15). Three coordinate systems are introduced, together with their basis vectors:

- The Cartesian coordinates $(C)$, with basis vectors $(\mathbf{i}, \mathbf{j}, \mathbf{k})$

- The spherical coordinates $(S)$, with basis vectors $\left(\mathbf{e}_{r}, \mathbf{e}_{\theta}, \mathbf{e}_{\varphi}\right)$

- The radial-orthradial-out-of-plane coordinates $(R)$, with basis vectors $\left(\mathbf{e}_{r}, \mathbf{e}_{\theta}, \mathbf{e}_{\varphi}\right)$

Written in $(C)$, the basis vectors of $(S)$ are:

$$
\begin{aligned}
\mathbf{e}_{r} & =\mid \begin{array}{c}
\cos \theta \cos \varphi \\
\sin \theta \cos \varphi \\
\sin \varphi
\end{array} \\
\mathbf{e}_{\theta} & =\mid \begin{array}{c}
-\sin \theta \\
\cos \theta \\
0
\end{array} \\
\mathbf{e}_{\varphi} & =\mathbf{e}_{r} \times \mathbf{e}_{\theta}=\mid \begin{array}{c}
-\cos \theta \sin \varphi \\
-\sin \theta \sin \varphi \\
\cos \varphi
\end{array}
\end{aligned}
$$

Therefore the matrix that transforms the coordinates of a vector in $(S)$ to the coordinates in $(C)$ is:

$$
P_{(S) \rightarrow(C)}=\left(\begin{array}{ccc}
\cos \theta \cos \varphi & -\sin \theta & -\cos \theta \sin \varphi \\
\sin \theta \cos \varphi & \cos \theta & -\sin \theta \sin \varphi \\
\sin \varphi & 0 & \cos \varphi
\end{array}\right)
$$

By differentiating the components of basis vectors of $(S)$, and using $P_{(C) \rightarrow(S)}=P_{(S) \rightarrow(C)}^{-1}=P_{(S) \rightarrow(C)}^{T}$, one obtains the expression of the derivatives of $\mathbf{e}_{r}, \mathbf{e}_{\theta}$ and $\mathbf{e}_{\varphi}$ with respect to $\theta$, expressed in $(S)$ : 


$$
\begin{aligned}
\frac{d \mathbf{e}_{r}}{d \theta} & =\mid \begin{array}{c}
0 \\
\cos \varphi \\
\varphi^{\prime} \\
(S)
\end{array} \\
\frac{d \mathbf{e}_{\theta}}{d \theta} & =\mid \begin{array}{c}
-\cos \varphi \\
0 \\
\sin \varphi_{(S)}
\end{array} \\
\frac{d \mathbf{e}_{\varphi}}{d \theta} & =\mid \begin{array}{c}
-\varphi^{\prime} \\
-\sin \varphi \\
0
\end{array}(S)
\end{aligned}
$$

The velocity vector $\tilde{\mathbf{v}}=\frac{d \mathbf{r}}{d \theta}$ can now be expressed in the spherical coordinates, knowing that $\mathbf{r}=r \mathbf{e}_{r}$ :

$$
\begin{aligned}
\tilde{\mathbf{v}} & =r^{\prime} \mathbf{e}_{r}+r \frac{d \mathbf{e}_{r}}{d \theta} \\
& =\mid \begin{array}{c}
r^{\prime} \\
r \cos \varphi \\
r \varphi^{\prime}
\end{array}(S)
\end{aligned}
$$

The out-of-plane basis vector is therefore:

$$
\begin{aligned}
\mathbf{e}_{h} & =\frac{\mathbf{e}_{r} \times \tilde{\mathbf{v}}}{\left\|\mathbf{e}_{r} \times \tilde{\mathbf{v}}\right\|} \\
& =\frac{1}{\sqrt{U}} \mid \begin{array}{c}
0 \\
-\varphi^{\prime} \\
\cos \varphi_{(S)}
\end{array}
\end{aligned}
$$

Where $U=\varphi^{\prime 2}+\cos ^{2} \varphi$. $\mathbf{e}_{o}$ is finally expressed in the spherical coordinates:

$$
\begin{aligned}
\mathbf{e}_{o} & =\mathbf{e}_{h} \times \mathbf{e}_{r} \\
& =\frac{1}{\sqrt{U}} \mid \begin{array}{c}
0 \\
\cos \varphi \\
\varphi^{\prime}
\end{array}
\end{aligned}
$$

The matrix that transforms the coordinates of a vector in $(R)$ to the coordinates in $(S)$ is then:

$$
P_{(R) \rightarrow(S)}=\left(\begin{array}{ccc}
1 & 0 & 0 \\
0 & \frac{\cos \varphi}{\sqrt{U}} & \frac{-\varphi^{\prime}}{\sqrt{U}} \\
0 & \frac{\varphi^{\prime}}{\sqrt{U}} & \frac{\cos \varphi}{\sqrt{U}}
\end{array}\right)
$$

Using $P_{(S) \rightarrow(R)}=P_{(R) \rightarrow(S)}^{-1}=P_{(R) \rightarrow(S)}^{T}$, the velocity vector can be expressed in $(R)$ : 


$$
\tilde{\mathbf{v}}=\mid \begin{gathered}
r^{\prime} \\
r \sqrt{U} \\
0
\end{gathered}
$$

From the latter, one can see that to get the components of $\tilde{\mathbf{a}}=\frac{d \tilde{\mathbf{v}}}{d \theta}$ in $(R)$, the expressions of $\frac{d \mathbf{e}_{r}}{d \theta}$ and $\frac{d \mathbf{e}_{o}}{d \theta}$ are required. $P_{(S) \rightarrow(R)}$ provides the components of $\frac{d \mathbf{e}_{r}}{d \theta}$ in $(R)$ :

$$
\frac{d \mathbf{e}_{r}}{d \theta}=\mid \begin{gathered}
0 \\
\sqrt{U} \\
0
\end{gathered}
$$

By differentiating $\mathbf{e}_{o}$ and using the expression of $\frac{d \mathbf{e}_{\theta}}{d \theta}$ and $\frac{d \mathbf{e}_{\varphi}}{d \theta}$ in spherical coordinates, one gets $\frac{d \mathbf{e}_{o}}{d \theta}$ in $(S)$ first:

$$
\frac{d \mathbf{e}_{o}}{d \theta}=\mid \begin{gathered}
-\sqrt{U} \\
-U^{-3 / 2} \varphi^{\prime}\left(\cos \varphi\left(\varphi^{\prime \prime}-\sin \varphi \cos \varphi\right)+2 U \sin \varphi\right) \\
U^{-3 / 2} \cos \varphi\left(\cos \varphi\left(\varphi^{\prime \prime}-\sin \varphi \cos \varphi\right)+2 U \sin \varphi\right)_{(S)}
\end{gathered}
$$

Then, with $P_{(S) \rightarrow(R)}$, the latter is expressed in $(R)$ :

$$
\frac{d \mathbf{e}_{o}}{d \theta}=\mid \begin{gathered}
-\sqrt{U} \\
0 \\
U^{-1}\left(\cos \varphi\left(\varphi^{\prime \prime}-\sin \varphi \cos \varphi\right)+2 U \sin \varphi\right)_{(R)}
\end{gathered}
$$

So finally $\mathbf{a}$ is obtained in $(R)$ :

$$
\tilde{\mathbf{a}}=\mid \begin{gathered}
r^{\prime \prime}-r U \\
2 r^{\prime} \sqrt{U}+r \varphi^{\prime} \frac{\varphi^{\prime \prime}-\sin \varphi \cos \varphi}{\sqrt{U}} \\
\frac{r}{\sqrt{U}}\left(\cos \varphi\left(\varphi^{\prime \prime}-\sin \varphi \cos \varphi\right)+2 U \sin \varphi\right)
\end{gathered}
$$

One can also check that in the spherical coordinate system, $\tilde{\mathbf{a}}$ is written:

$$
\tilde{\mathbf{a}}=\mid \begin{gathered}
r^{\prime \prime}-r U \\
2 r^{\prime} \cos \varphi-2 r \varphi^{\prime} \sin \varphi \\
2 r^{\prime} \varphi^{\prime}+r\left(\varphi^{\prime \prime}+\sin \varphi \cos \varphi\right)_{(S)}
\end{gathered}
$$

The expression of $\tilde{\mathbf{a}}$ in Cartesian coordinates is obtained by applying $P_{(S) \rightarrow(C)}$ to the latter. 


\section{Acknowledgments}

The work presented in this paper is partially supported by Qinetiq through an ICASE EPSRC grant. The authors would like to thank Dr. Michael Efroimsky and Dr. Bradley Wall whose useful and interesting suggestions contributed to the improvement of this paper.

\section{References}

[1] Rayman, M.D., Varghese, P., Lehman, D.H. and Livesay, L.L.: Results from the Deep Space 1 Technology Validation mission, Acta Astronautica, Issues 2-9, July-November 2000, pp. 475-487

[2] Haros, D. and Schoenmakers, J.:Post-Launch Optimisation of the Smart 1 Low-Thrust Trajectory to the Moon, 18th International Symposium on Spaceflight Dynamics, Munich, Germany, 15th-22nd Oct. 2004

[3] Russel, C.T., Capaccioni, F., Corradini, A., Christensen, U., De Sanctis M.C., Feldman W.C., Jaumann R., Keller H.U., Konopliv A., McCord T.B., McFadden L.A., McSween H.Y., Mottola S., Neukum G., Pieters C.M., Prettyman T.H., Raymond C.A., Smith D.E., Sykes M.V., Williams B. and Zuber M.T. Dawn Discovery Mission to Vesta and Ceres: Present Status. Advances in Space Research, Vo. 38, Issue 9, 2006, pp 2043-2048

[4] Kuninaka H., Nishiyama K., Funaki I., Yamada T., Shimizu Y. Powered Flight of HAYABUSA in Deep Space. 42nd AIAA/ASME/SAE/ASEE Joint Propulsion Conference \& Exhibit 9-12 July 2006, Sacramento, California

[5] Yarnoz D.G., Jehn R., De Pascale P. Trajectory Design for Bepi-Colombo Mission to Mercury. Journal of the British Interplanetary Society, JBIS, June 2007

[6] Betts, J.T.: Survey of Numerical Methods for Trajectory Optimization, Journal of Guidance, Control and Dynamics, Vol. 21, No. 2, March-April 1998, pp. 193-207

[7] Markopoulos, N., Non-Keplerian manifestations of the Keplerian trajectory equation and a theory of orbital motion under continuous thrust, AAS Spaceflight Mechanics conference, pp. 1061-1080, 2005

[8] Petropoulos, A.E. and Longuski, J.M.: Shape-Based Algorithm for the Automated Design of Low-Thrust, Gravity-Assist Trajectories, Journal of Spacecraft and Rockets, Vol. 41, No. 5, September-October 2004, pp. 787-796

[9] Wall, B.J. and Conway, B.A.: Shape-Based Approach to Low-Thrust Rendezvous Trajectory Design, Journal of Guidance, Control and Dynamics, Vol. 32, No. 1, January-February 2009, pp. 95-101.

[10] Wall B. Shape-Based Approximation Method for Low-Thrust Trajectory Optimization. AIAA-2008-6616, AIAA/AAS Astrodynamics Specialist Conference and Exhibit, Honolulu, Hawaii, August 18-21, 2008.

[11] De Pascale P., Vasile M., Preliminary Design of Low-Thrust Multiple Gravity Assist Trajectories. Journal of Spacecraft and Rockets, Vol. 43, Number 5, September-October 2006.

[12] Vasile, M., De Pascale, P. and Casotto, S.: On the optimality of a shape-based approach based on pseudo-equinoctial elements, Acta Astronautica, Volume 61, Issues 1-6, June-August 2007, pp. $286-297$.

[13] Lane, S.H. and Stengel, R.F.: Flight Control Design Using Non-linear Inverse Dynamics, Autonomia, Vol. 24, No. 4, pp. 471-83.

[14] Baumgarte, J.: Stabilization of constraints and integrals of motion in dynamical systems, Computer methods in applied mechanics and engineering, Elsevier, Vol. 1, No. 1, pp 1-16, 1972

[15] Vasile, M., Bernelli-Zazzera F.: Optimizing Low-Thrust and Gravity Assist Maneuvres to Design Interplanetary Trajectories, The Journal of the Astronautical Sciences. Vol. 51, No. 1, January-March 2003. 
[16] Battin, R. H., An introduction to the mathematics and methods of astrodynamics, AIAA Education Series, 1999, pp. 490493

[17] Petropoulos, A.E. and Longuski, J.M.: Automated design of low-thrust gravity-assist trajectories, AIAA/AAS Astrodynamics Specialist Conference, 2000, Vol. 4033, pp. 157-166

[18] Petropoulos, A.E. and Longuski, J.M.: Shape-based algorithm for automated design of low-thrust, gravity-assist trajectories, Journal of Spacecraft and Rockets, Vol. 41, No. 5, 2004, pp. 787-796

[19] Izzo, D.: Lambert's problem for exponential sinusoids, Journal of Guidance, Control and Dynamics, Vol. 29, No. 5, 2005, pp. $1242-1245$

[20] Efroimsky, M.: Gauge Freedom in Astrodynamics, Modern Astrodynamics, edited by P. Gurfil, Butterworth-Heinemann, 2006, pp. 23-52.

[21] McConaghy, T.T., Debban, T.J., Petropoulos, A.E. and Longuski, J.M.: Design and optimization of low-thrust trajectories with gravity assists, Journal of spacecraft and rockets, Vol. 40, No. 3, 2003, pp. 380-387 